ARTICLE

DOI: $10.1038 / s 41467-017-01864-y$

\title{
Discovery of naturally occurring ESR1 mutations in breast cancer cell lines modelling endocrine resistance
}

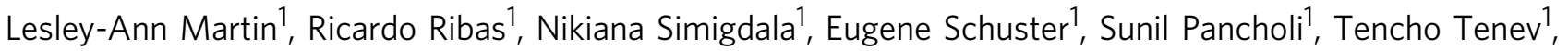
Pascal Gellert ${ }^{1}$, Laki Buluwela ${ }^{2}$, Alison Harrod², Allan Thornhill³, Joanna Nikitorowicz-Buniak1, Amandeep Bhamra ${ }^{4}$, Marc-Olivier Turgeon ${ }^{5}$, George Poulogiannis ${ }^{5,6}$, Qiong Gao ${ }^{1}$, Vera Martins ${ }^{7}$, Margaret Hills ${ }^{7}$, Isaac Garcia-Murillas ${ }^{1}$, Charlotte Fribbens ${ }^{1}$, Neill Patani ${ }^{1}$, Zheqi Li ${ }^{8}$, Matthew J. Sikora ${ }^{8}$, Nicholas Turner ${ }^{1}$, Wilbert Zwart ${ }^{9}$, Steffi Oesterreich (i) ${ }^{8}$, Jason Carroll ${ }^{10}$, Simak Ali id ${ }^{2} \&$ Mitch Dowsett $^{1,7}$

Resistance to endocrine therapy remains a major clinical problem in breast cancer. Genetic studies highlight the potential role of estrogen receptor- $\alpha$ (ESR1) mutations, which show increased prevalence in the metastatic, endocrine-resistant setting. No naturally occurring ESR1 mutations have been reported in in vitro models of BC either before or after the acquisition of endocrine resistance making functional consequences difficult to study. We report the first discovery of naturally occurring ESR1 ${ }^{Y 537 C}$ and ESRT ${ }^{Y 537 S}$ mutations in MCF7 and SUM44 ESR1-positive cell lines after acquisition of resistance to long-term-estrogendeprivation (LTED) and subsequent resistance to fulvestrant (ICIR). Mutations were enriched with time, impacted on ESR1 binding to the genome and altered the ESR1 interactome. The results highlight the importance and functional consequence of these mutations and provide an important resource for studying endocrine resistance.

\footnotetext{
${ }^{1}$ Breast Cancer Now Toby Robins Research Centre, Institute of Cancer Research, London SW7 3RP, UK. ${ }^{2}$ Division of Cancer, CRUK Labs, University of London Imperial College, London W12 ONN, UK. ${ }^{3}$ Centre for Cancer Imaging, Institute of Cancer Research, Sutton SM2 5NG, UK. 4 Proteomic Unit, Institute of Cancer Research, London SW7 3RP, UK. ${ }^{5}$ Division of Cancer Biology, The Institute of Cancer Research, London SW3 6JB, UK. ${ }^{6}$ Division of Computational and Systems Medicine, Department of Surgery and Cancer, Imperial College London, London SW7 2AZ, UK. ${ }^{7}$ Ralph Lauren Centre for Breast Cancer Research, Royal Marsden Hospital, London SW3 6JB, UK. ${ }^{8}$ Department of Pharmacology and Chemical biology, University of Pittsburgh, Pittsburgh, PA 15213, USA. ${ }^{9}$ Department of Molecular Pathology, Netherlands Cancer Institute, 1066CX Amsterdam, Netherlands. ${ }^{10}$ Cancer Research UK Cambridge Institute, University of Cambridge, Cambridge CB2 ORE, UK. Lesley-Ann Martin, Ricardo Ribas, Nikiana Simigdala and Eugene Schuster contributed equally to this work. Correspondence and requests for materials should be addressed to L.-A.M. (email: Lesley-ann.martin@icr.ac.uk)
} 
ver $70 \%$ of breast cancers (BC) are estrogen receptor- $\alpha$ (ESR1) positive at diagnosis. Estrogen mediates its effects by binding to ESR1 leading to expression of genes controlling proliferation and cell survival. ESR1 has two distinct activation domains, AF-1 and AF-2. AF-1 is regulated by phosphorylation while AF-2 is integral to the ligand-binding domain (LBD) and associates with coactivators, controlling the ESR1 transcriptional complex (reviewed by Green and Carroll ${ }^{1}$ ). Classically, patients with ESR1-positive BC are treated with endocrine agents such as tamoxifen, aromatase inhibitors (AIs), or fulvestrant, which impede ESR1-signaling (reviewed by Ma et al. ${ }^{2}$ ). Although over $50 \%$ of ESR1-positive patients show response to endocrine therapy and estrogen deprivation therapy reduces $\mathrm{BC}$ mortality by $40 \%^{3}$, a large proportion relapse with de novo or acquired resistant disease, making it one of the greatest challenges for $\mathrm{BC}$ research and treatment.

Multiple mechanisms of resistance have been proposed, most of which have been identified using a limited number of ESR1positive $\mathrm{BC}$ cell lines. These include aberrant cross-talk between ESR1 and growth factor signaling pathways or alterations in the balance of coactivators and corepressors (reviewed by Ma et al. ${ }^{2}$, Osborne et al. ${ }^{4}$, and Miller et al..$^{5}$ ).

It has been known for many years that some mutations in ESR1 can lead to ligand-independent activation, but until recently, such mutations appeared to have little clinical significance ${ }^{6}$, as their presence in primary disease is rare. However, the prevalence of ESR1 mutations in metastatic tumors that have recurred or progressed after endocrine therapy is far higher ${ }^{7-9}$. We have recently reported that the detection of these mutations in circulating tumor DNA (ctDNA) of $39.1 \%$ of metastatic patients appears to correlate with clinical resistance to $\mathrm{AIs}^{10}$. The majority of ESR1 mutations are located at two amino acids in the LBD Y537N/C/S and D538G. Functional studies using ectopic expression of these mutations led to constitutive activity of ESR1 and conferred partial resistance to established clinical doses of tamoxifen and fulvestrant ${ }^{11,12}$. However, as these mutations were engineered, the role of cellular context during acquisition of resistance with time was not explored.

In this manuscript, we report for the first time, the identification of naturally occurring ESR1 mutations in BC cell models and their enrichment during acquisition of resistance to endocrine therapy. We show that the mutated ESR1 controls a cistrome similar to the ligand-dependent wt ESR1 and associates with an altered protein interactome enabling ligand-independent proliferation. Furthermore, these naturally occurring ESR1 mutants are sensitive to fulvestrant, suggesting that this and similar agents may have applicability in patients with tumors harboring these mutations supporting our recent clinical data ${ }^{13}$.

\section{Results}

Discovery of ESR1 mutations in models of endocrine resistance. Previously, we reported the development of long-termestrogen-deprived (LTED) derivatives from a number of ESR1positive BC cell lines (including MCF7, HCC1428, T47D, ZR75.1, and SUM44) $)^{14,15}$. In general, estrogen deprivation leads to an initial quiescent population accompanied by cell death and after many weeks to outgrowth of a cell population that then proliferates independently of exogenous estrogen (Supplementary Fig. 1a-d). The phenotype of the LTED cell lines varies leading to a context-specific sensitivity or resistance to additional agents ${ }^{14}$.

As ESR1 mutations have been associated with resistance to endocrine therapy, we explored whether these mutations or those of other genes were either enriched or acquired in the in vitro models described. Whole-exome sequencing from wt-MCF7 and MCF7-LTED showed an ESR1 ${ }^{\mathrm{Y} 537 \mathrm{C}}$ mutation in the MCF7-
LTED at an estimated variant allele frequency (VAF) of $30 \%$, while it was undetectable in the wt-MCF7. The mutation was validated using digital droplet (dd) PCR (Fig. 1a, b).

ESR1 mutations occur in LTED but not tamoxifen-resistant cells. As a result of this unexpected finding, we sequenced known hotspot regions for ESR $1^{16}$ by Ion Torrent in wt and LTED derivatives of MCF7, SUM44, HCC1428, and ZR75.1, together with tamoxifen-resistant (TAMR) derivatives of MCF7 and HCC1428 and fulvestrant-resistant (ICIR) derivatives of wtMCF7, MCF7-LTED, and ZR75.1-LTED (Table 1; Supplementary Fig. 2). The ESR1 ${ }^{\text {Y537C }}$ mutant was detected in the MCF7-LTEDICIR cells at a VAF of $48 \%$ that was confirmed by ddPCR (49.8\%) (Supplementary Fig. 3a) but was not detected in the wt-MCF7ICIR cells. Comparison of the two isogenic models showed that fulvestrant resistance (Supplementary Fig. 3b) occurred irrespective of the mutation. Furthermore, both ICIR derivatives showed a marked reduction in ESR1 (Supplementary Fig. 3c) and a concomitant drop in expression of estrogen-regulated genes (GREB1, PDZK1, PGR, and TFF1) but equivalent expression of genes associated with proliferation when compared to their respective wt (Supplementary Fig. 3d).

Strikingly, analysis by Ion torrent also revealed an ESR $1^{\text {Y537S }}$ heterozygous mutation in SUM44-LTED (VAF 47\%). ESR1 mutations were confirmed by Sanger sequencing, RNA sequencing, mass spectrometry, and whole-exome sequencing (Supplementary Fig. 4a-g). Exome sequencing did not reveal any additional mutated genes involved in AI resistance beyond the mutation in ESR1 nor did it reveal mutations in genes known to be drivers of $\mathrm{BC}^{17}$ that might promote growth by other mechanisms (Supplementary Data 1).

In order to determine if the ESR1 $1^{Y 537 C}$ VAF of $30 \%$ in the MCF7-LTED cells was indicative of a mixed population of cells harboring either ESR $1^{\text {wt }}$ or ESR $1^{Y 537 C}$, we assessed ESR1 copy number by fluorescent in situ hybridization (FISH) and exome sequencing. This revealed an allelic imbalance, which on average identified two or more wt copies of ESR1 and one mutant copy per cell in the MCF7-LTED, indicating $100 \%$ of the cell population harbored the mutation. In contrast, the MCF7LTED-ICIR cells were enriched for two copies of ESR1 per cell similar to the SUM44-LTED, accounting for the VAF of $50 \%$ again indicating every cell in the given population contained a mutation (Supplementary Fig. 5).

Temporal enrichment of ESR1 mutations during estrogen deprivation. Analysis by ddPCR over a time course showed that the ESR1 ${ }^{\text {Y537S }}$ mutation was detectable within 12 weeks following transfer of SUM44 cells to estrogen-free medium (Fig. 1c). Thereafter, the VAF increased progressively up to $50 \%$. In order to determine if the mutation was present in the parental population or was acquired as a result of the selective pressure of estrogen withdrawal, we screened over $6 \times 10^{6}$ matched parental SUM44 copies. Interestingly, the ESR $1^{\text {Y537S }}$ mutation was present in wt-SUM44 at an apparent frequency of $\sim 1: 1.000 .000$ (Fig. 1d), implying that the ESR1 $1^{Y 537 S}$ mutation pre-exists in a very small proportion of SUM44 cells. We further screened a second batch of SUM44-LTED and their corresponding parent cell line ${ }^{18}$ but no mutation was identified, suggesting this is not the only adaptive mechanism. In order to control further the potential of contamination, we screened an equivalent number of ESR1negative SKBR3 cells and no mutation was evident (Fig. 1d). Finally, to address the possibility that the Y537C mutation was also resident at low frequency in MCF7 cells, we screened three independent batches, covering over $6 \times 10^{6}$ copies, however we were unable to identify the Y537C mutation. 
a

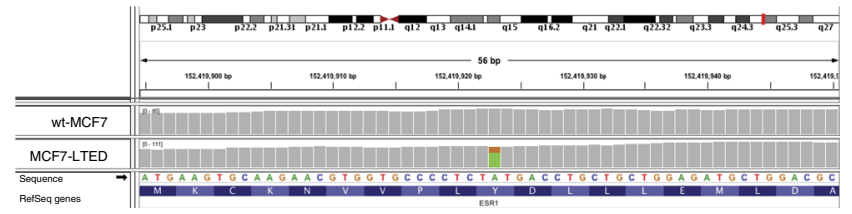

b

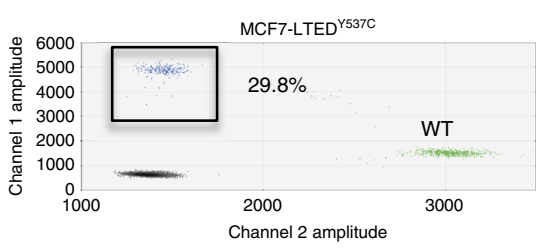

d
C
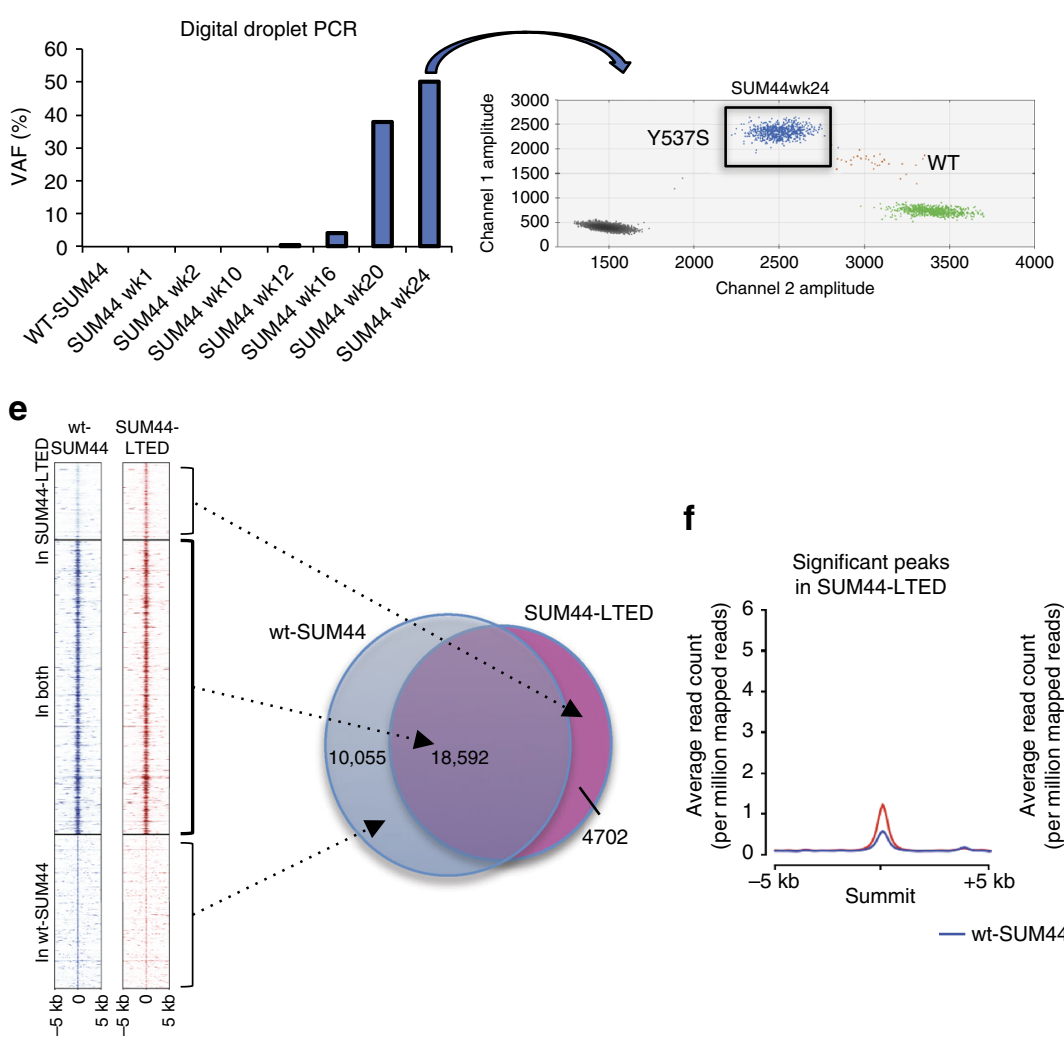
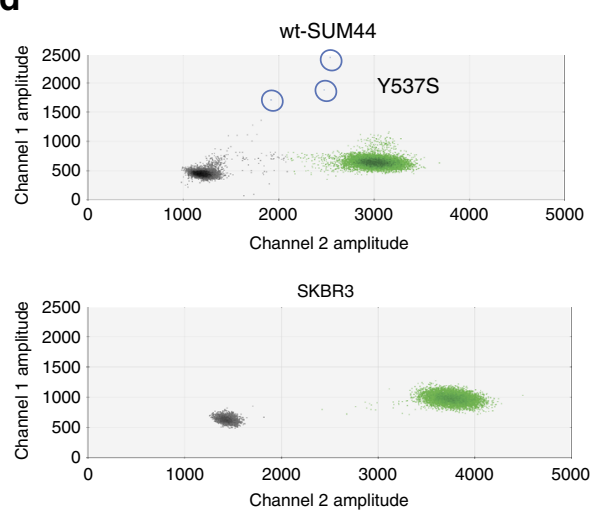
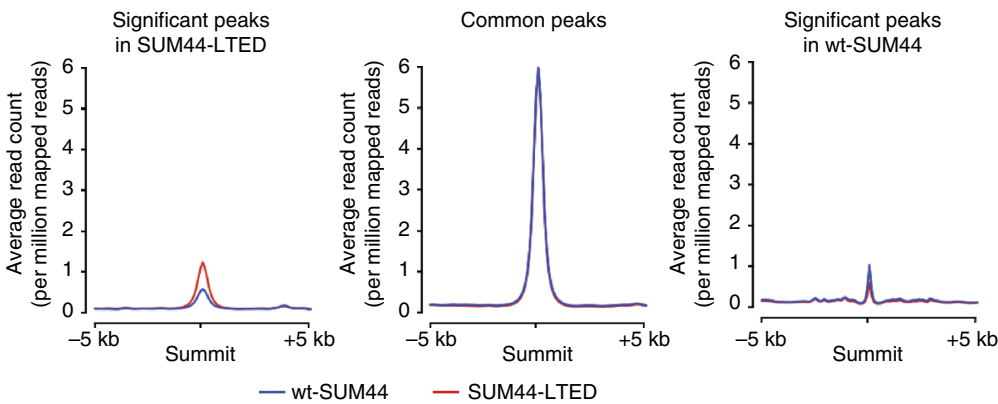

g

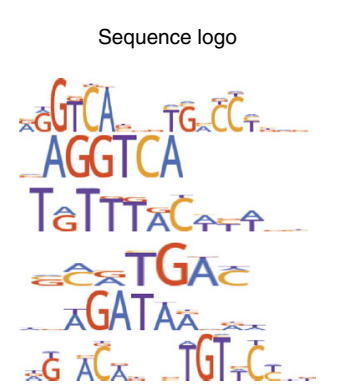

Name
ESR1
RARA
FOXA1
PAX2
GATA3
ANDR

wt-SUM44
$10^{-72}$
$10^{-48}$
$10^{-21}$
$10^{-4}$
$10^{-58}$
$10^{-5}$

p-value

h

Enrichment plot:

Induced binding events in LTED

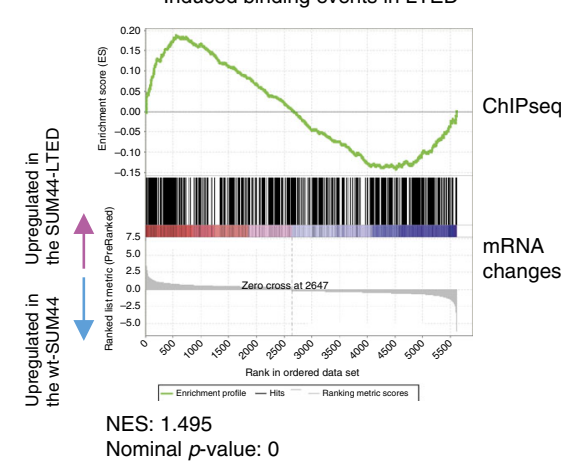

Fig. 1 Identification and characterization of ESR1 mutations in models of endocrine resistance. a Visualization of ESR1 ${ }^{Y 537 C}$ identified during exome sequencing. b Digital droplet PCR (ddPCR) showing the presence of the ESRT ${ }^{Y 537 C}$ mutation in MCF7-LTED. c ddPCR showing the presence of the ESR7 ${ }^{Y 537 S}$ mutation in SUM44-LTED. Temporal analysis showing enrichment of the mutation from wk12 post-estrogen deprivation. $\mathbf{d}$ ddPCR showing the presence of ESR1 ${ }^{\text {Y537S }}$ at low variant allele frequency (VAF) in wt-SUM44 but not in SKBR3. e Overlap between wt-SUM44 and SUM44-LTED ESR1 binding sites and corresponding heatmap. The heatmap depicts binding peak intensities, which are common or different between the two cell lines. The window represents $\pm 5 \mathrm{~kb}$ regions from the center of the binding event. $\mathbf{f}$ Comparison of the average read count between wt-SUM44 and SUM44-LTED showing peak affinity for the common and different binding events between the two cell lines. $\mathbf{g}$ Motif analysis of common and augmented ESR1 peaks from wt-SUM44 vs. SUM44-LTED. $p$-value of "common peaks" based on average of three random selections of 2150 peaks to approximately match the number of peaks within the "augmented peak" comparisons. h GSEA was conducted comparing RNA-seq with ESR1-induced binding events in SUM44-LTED. ChIP-seq analysis was carried out using data from two biological replicates and RNA-seq from three biological replicates 
Table 1 Identification of naturally occurring ESR1 mutations in cell line models of endocrine sensitive and resistant breast cancer

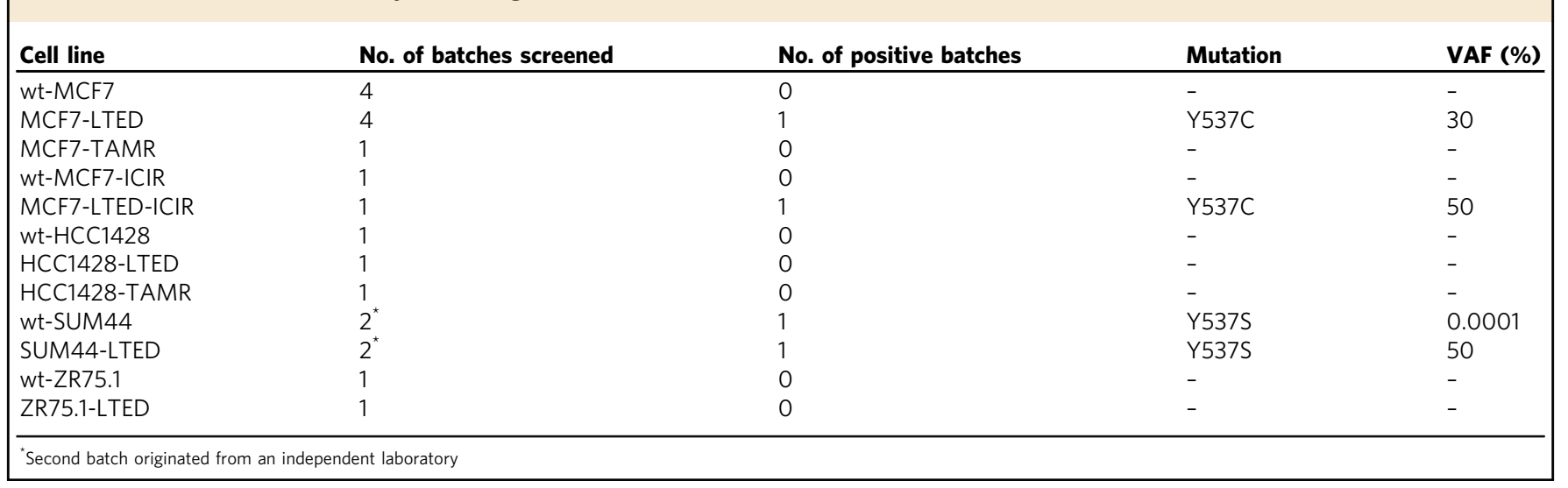

ESR1 ${ }^{\text {Y537S }}$ drives ligand-independent transcription. To determine the function of ESR1 ${ }^{Y 537 S}$, we performed ChIP-seq with antibodies for ESR1 in asynchronous wt-SUM44 in the presence of estrogen and SUM44-LTED in the absence of estrogen. Overlap of two replicate experiments called 28,647 and 23,294 ESR1 binding events in wt-SUM44 and SUM44-LTED cells, respectively. The vast majority $(80 \%)$ of the ESR1 ${ }^{\mathrm{Y} 537 \mathrm{~S}}$ binding sites in SUM44-LTED cells were common to ESR ${ }^{\text {wt }}$ binding sites in estrogen-treated wt-SUM44 (Fig. 1e). Although 4702 differential binding sites were called in the SUM44-LTED cells, these were not unique, but represented enriched ESR1 binding, i.e., they also appeared in wt-SUM44 and this was similarly the case for the 10,055 differential binding sites in wt-SUM44 that occurred in the SUM44-LTED but were not enriched to the same level (Fig. 1f).

Peak strength was evaluated at a number of target genes (Supplementary Fig. 6a), where augmented ESR1 ${ }^{\mathrm{Y} 5375}$ binding was evident in SUM44-LTED compared to wt-SUM44. Furthermore, ChIP-qPCR validation assessing recruitment of ESR1 ${ }^{\mathrm{Y} 537 \mathrm{~S}}$ together with FOXA1, a major pioneer factor for ESR $1^{19}$ and CBP required for an authentic ESR1 transcriptional complex ${ }^{20}$, showed enhanced binding to the promoters of TFF1 and GREB1 in the SUM44-LTED compared to wt cell line (Supplementary Fig. 6b).

ESR1 binding sites in both cell lines showed a similar pattern of genomic distribution (Supplementary Fig. 6c). Furthermore, the vast majority of binding motifs were similar for ESR1 ${ }^{\mathrm{wt}}$ and ESR1 ${ }^{\text {Y537S }}$, however, significant enrichment for motifs representing the transcription factors ESR1, RARA, PAX2, ANDR, and FOXA1 were evident in relation to the enriched ESR1 peaks found in SUM44-LTED, compared to wt-SUM44, which conversely showed increased GATA3 (Fig. 1g).

To identify the transcription targets of $\mathrm{ESR}^{\mathrm{Y} 537 \mathrm{~S}}$, we integrated ChIP-seq and RNA-seq data from the respective cell lines. Gene set enrichment analysis (GSEA) showed that increased ESR $1^{\mathrm{Y} 537 \mathrm{~S}}$ genomic binding correlated with increased transcription, whereas loss of binding correlated with downregulation of genes in SUM44-LTED (Fig. 1h; Supplementary Fig. 6d). We next used K-means clustering to compare the ESR1 binding patterns with expression of genes in wt-SUM44, wtSUM44 after 1 week of estrogen deprivation and the SUM44LTED (20 weeks of estrogen deprivation). We identified four distinct gene sets ${ }^{17}$ (Fig. 2a-c): GS1 consisted of classical estrogen-regulated genes such as TFF1, GREB1, PGR, and CCND1, which decreased in expression after 1 week of deprivation but were elevated in the SUM44-LTED. GS4 contained genes such as FOXA1 that were enriched after the first week of estrogen deprivation and remained active in the
LTED. GS2 and 3 included genes, such as MYC and JUN, which were downregulated in the SUM44-LTED compared to wtSUM44. Pathway analysis of the four clusters showed enrichment of ESR1 signaling, epithelial-to-mesenchymal transition (EMT), mTORC1 complex activation, and cholesterol homeostasis in the SUM44-LTED.

To address this further, we assessed the metabolic capability of the wt-SUM44 and SUM44-LTED using Seahorse (Fig. 2d). No significant change in glutamine dependency was evident between the two cell lines; however, the SUM44-LTED showed a significantly higher glutamine capacity and fatty acid dependency compared to the wt-SUM44. The SUM44-LTED also showed a slight but significant decrease in glucose dependency.

Finally, we assessed the migratory ability of the cell lines (Fig. 2e). The SUM44-LTED showed a two-fold increase $(p<$ 0.001 , Student's $t$ test) in migration compared to wt-SUM44.

Collectively, these findings suggest that ESR1 ${ }^{\text {Y537S }}$ mediates binding events that are functionally significant and lead to expression of genes controlling proliferation, survival and EMT, in a ligand-independent manner and while many ESR1 binding events are similar between the two lines, differences do exist and are probably the result of, or influenced by, the cellular context.

ESR1 ${ }^{\mathrm{Y} 537 \mathrm{~S}}$ interacts with known ESR1 binding proteins. In order to elucidate the impact of the Y537S mutation on the ESR1 interactome and proteome, we carried out comparative RIME (rapid immunoprecipitation with tandem mass spectrometry of endogenous proteins) and dimethyl labeling ${ }^{21}$ between wtSUM44 and SUM44-LTED (Fig. 3a; Supplementary Fig. 7a, b). RIME demonstrated ESR $1{ }^{\text {Y537S }}$ associated with a similar portfolio of proteins to those seen for ESR1 ${ }^{\text {wt }}$ including ESR1 itself, as well as, PGR, TLE3, HAT1, and FOXA1 ${ }^{22}$. However, increased association between ESR1 ${ }^{\text {Y537S }}$ GREB1 and FOXA1 was noted, which we confirmed by Co-IP (Supplementary Fig. 7c). Quantitation of proteins by dimethyl labeling showed increased abundance of TFF1 and a slight increase in ESR1 but not FOXA1 (Supplementary Fig. 7d).

Immunoblot analysis of wt-SUM44 and SUM44-LTED under basal growth conditions was assessed for changes in growth factor receptors and down stream pathways associated with endocrine resistance $^{2}$ as well as alterations in $\mathrm{pESR} 1^{\text {ser118}}, \mathrm{pESR} 1^{\text {ser167, and }}$ PGR (Fig. 3b; Supplementary Fig. 8). No significant changes in pEGFR or pERBB2 were apparent between the cell lines. A slight increase in $\mathrm{pERK} 1 / 2$ was seen in SUM44-LTED but no change in pAKT ${ }^{\text {ser473}}$. The level of pESR $1^{\text {ser118 }}$ was greater in wt-SUM44 compared to the SUM44-LTED. However, a slight increase in 
pESR $1^{\text {ser167 }}$ was noted in the LTED model (Fig. 3b). To address this further, both wt-SUM44 and SUM44-LTED were cultured in DCC medium in the absence or presence of estrogen. In this setting, ESR1 abundance and phosphorylation profiles were similar between the SUM44-LTED in the absence of estrogen and the wt-SUM44 in the presence of estrogen. Overall, these data showed the profile of the wt-SUM44 and SUM44-LTED were similar (Supplementary Fig. 7e). a

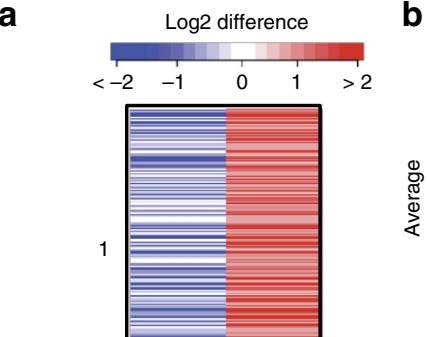

b
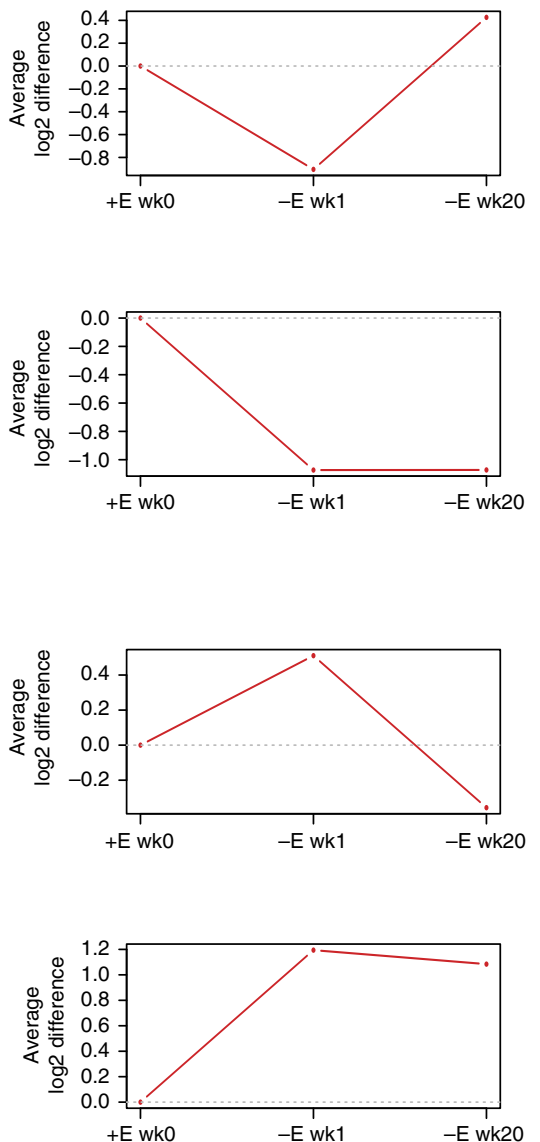

d

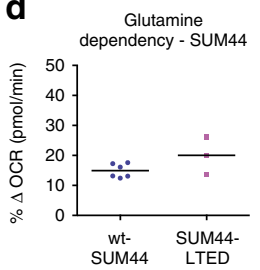

Fatty acid

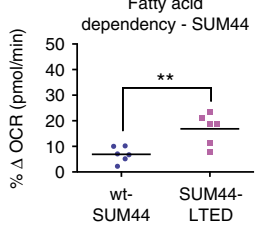

Glucose dependency - SUM44

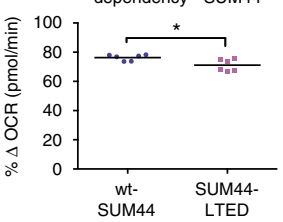

Gene set name

HALLMARK_ESTROGEN_RESPONSE_EARLY

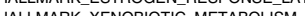

HALMARK_XENOBOTC_META

HALLMARK EPITHELIAL MESENCHYMAL TRANSITION -

HALLMARK_TNFA_SIGNALING_VIA_NFKB

HALLMARK GLYCOLYSIS

HALLMARK_KRAS SIGNALING_UP

HALLMARK_INTERFERON_GAMMA_RESPONSE

HALLMARK_MTORC1_SIGNALING

HALLMARK_MYOGENESIS

HALLMARK_FATTY_ACID_METABOLISM

Gene set name

HALLMARK_TNFA_SIGNALING_VIA-NFKB

HALLMARK_ESTRIGEN_RESPONSE_EARLY

HALLMARK_MTORC1_SIGNALING

HALLMARK_UNFOLDED_PROTEIN_RESPONSE

HALLMARK_ESTROGEN_RESPONSE_LATE

HALLMARK_HYPOXIA

HALLMARK_P53_PATHWAY

ATHAY

HALIMARK GLYCOLYSIS

HALLMARK III STAT5 SIGNALING

HALLMARK XENOBIOTIC METABOLISM

HALLMARK UV RESPONSE UP

HALLMARK_INTERFERON_GAMMA RESPONSE

HALLMARK_CHOLESTEROL_HOMEOSTASIS

Gene set name

HALLMARK_MITOTIC_SPINDLE

HALLMARK_G2M_CHECKPOINT

HALLMARK_P53_PATHWAY

HALLMARK_HYPOXIA

HALLMARK IL2 STAT5 SIGNALING

HALLMARK_TNFA_SIGNALING_VIA_NFKB

HALLMARK_ADIPOGENESIS

HALLMARK_APICAL_JUNCTION

HALLMARK_HEME_METABOLISM

HALLMARK_ANDROGEN_RESPONSE

HALLMARK_APOPTOSIS

HALLMARK_GLYCOLYSIS

HALLMARK_KRAS_SIGNALING_UP

Gene set name

HALLMARK_MTORC1_SIGNALING

HALLMARK_XENOBIOTIC_METABOLISM

HALLMARK_E2F_TARGETS

HALMARK HYPOXIA

HALLMARK PEROXISOME

HALLMARK UV RESPONSE DN

HALLMARK G2M CHECKPOINT

HALLMARK BILE_AICD METABOLISM

HALLMARK_INFLAMMATORY_RESPONSE

HALLMARK_KRAS_SIGNALING_UP

HALLMARK_MYOGENESIS

HALLMARK_CHOLESTEROL_HOMEOSTATIS

HALLMARK_APICAL_JUNCTION

HALUAR_KRAS_IG

FDR $q$-value

1.37E-42

$.95 \mathrm{E}-09$

$7.58 \mathrm{E}-07$

$7.58 \mathrm{E}-07$

$3.90 \mathrm{E}-06$

$3.90 \mathrm{E}-06$

$1.55 \mathrm{E}-05$

$1.95 \mathrm{E}-05$

9.29E-05

9.29E-05

3.35E-04

FDR $q$-value

3.49E-23

$4.59 \mathrm{E}-21$

$6.79 \mathrm{E}-19$

$1.31 \mathrm{E}-13$

$1.31 \mathrm{E}-13$

$1.21 \mathrm{E}-11$

1.00E-10

$5.60 \mathrm{E}-10$

4.36E-08

$4.36 \mathrm{E}-08$
$2.70 \mathrm{E}-07$

$2.70 \mathrm{E}-07$

$8.99 \mathrm{E}-07$

$8.99 \mathrm{E}-07$
$1.60 \mathrm{E}-06$

$5.76 \mathrm{E}-06$

FDR $q$-value

1.05E-24

2.69E-17

1.15E-10

1.23E-07

$1.23 \mathrm{E}-07$
$1.23 \mathrm{E}-07$

$1.23 \mathrm{E}-07$

$1.23 \mathrm{E}-07$
$1.99 \mathrm{E}-05$

$7.86 \mathrm{E}-05$

$7.86 \mathrm{E}-05$

$7.86 \mathrm{E}-05$
$1.25 \mathrm{E}-04$

$1.85 \mathrm{E}-04$

$2.76 \mathrm{E}-04$

$1.08 \mathrm{E}-03$

$1.39 \mathrm{E}-03$

FDR $q$-value

2.75E-07

$2.75 \mathrm{E}-07$

7.96E-06

$7.96 \mathrm{E}-06$

$1.24 \mathrm{E}-05$

$3.14 \mathrm{E}-05$

$3.14 \mathrm{E}-05$
$8.54 \mathrm{E}-05$

1.23E-04

1.23E-04

1.23E-04

3.40E-04

$4.92 \mathrm{E}-04$

4.92E-04

e

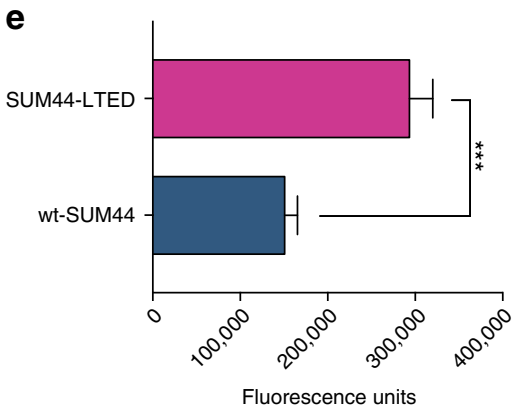


As FOXA1 is an important pioneer factor regulating ESR1driven transcription ${ }^{23}$, and FOXA1 sites were enriched in our ChIP-seq analysis of SUM44-LTED cells, we hypothesized that it played a pivotal role in transcriptional regulation of ESR1 ${ }^{\mathrm{Y} 537 \mathrm{~S}}$. Small interfering RNA (siRNA) knockdown of FOXA1 significantly reduced proliferation of both wt-SUM44 (42\%, $p<0.001$, Student's $t$ test) and SUM44-LTED cells although this was more pronounced in the latter $(75 \%, p<$ 0.001 , Student's $t$ test) (Fig. $3 \mathrm{c}$ ). siFOXA1 also correlated with a significant reduction in the expression of TFF1 and CCND1 (Fig. 3d), suggesting FOXA1 plays a crucial role in the ligandindependent transcriptional activity of ESR1 ${ }^{\mathrm{Y} 537 \mathrm{~S}}$.

\section{CRISPR analysis shows ESR1 ${ }^{\text {Y537S }}$ controls ligand indepen-} dence. As kinase signaling has been strongly implicated in endocrine resistance resulting in ligand-independent activity of $E S R 1^{2}$, we sought an approach that would reduce the effect of this confounding influence. In this setting, wt-MCF7, which harbor $E S R 1^{w t}$, were engineered to introduce the ESR1 ${ }^{Y 537 S}$ mutation using CRISPR-Cas9 genome editing. MCF7 ${ }^{\mathrm{Y} 537 \mathrm{~S}}$ cells carry one endogenous ESR1 gene in which, ESR $1^{\text {wt }}$ has been mutated to code for the ESR1 $1^{\text {Y537S }}$ mutation, as well as ESR1 ${ }^{\text {wt }}$. Detailed functional analyses of MCF7 ${ }^{\mathrm{Y} 537 \mathrm{~S}}$ cells are described elsewhere ${ }^{24}$. Proliferation assays in the absence of exogenous estrogen showed the MCF7 ${ }^{\mathrm{Y} 537 \mathrm{~S}}$ was ligand-independent (Fig. 4a). Furthermore, levels of ESR1 expression between the wt and the mutated cell line were similar (Fig. 4b; Supplementary Fig. 8). Analysis of ESR1 ChIP-seq from wt-MCF7 and MCF7 ${ }^{\text {537S }}$ in the absence of exogenous estrogen showed 3602 common peaks across the genome and 8094 unique binding events in MCF7 ${ }^{\mathrm{Y} 537 \mathrm{~S}}$ (Fig. 4c, d). Furthermore, peak affinity was greater for ESR1 1 Y537S across the genome while binding events were similarly distributed for both ESR1 $1^{\text {wt }}$ and ESR $1^{\text {Y537S }}$ (Fig. 4e, f). Overlay of the binding events from ChIP-seq analysis with corresponding RNA-seq from MCF7 ${ }^{\mathrm{Y} 537 \mathrm{~S}}$ showed increased expression of proliferationassociated genes and known estrogen-regulated genes, which was confirmed by protein expression (Fig. $4 \mathrm{~b}$, g; Supplementary Fig. 8). This data suggest the mutation alone is sufficient to hold ESR1 in a conformation suitable for recruitment of coactivators together with the basal transcription machinery and that these mutations may not require altered kinase profiles to be active. Of note, treatment of both cell lines with estrogen revealed $74 \%$ concordance in ESR1 binding events suggesting ESR1 ${ }^{\mathrm{Y} 537 \mathrm{~S}}$ remained responsive to ligand (Fig. $4 \mathrm{~h}$ ).

Intersect of the ESR1 binding events in SUM44-LTED ${ }^{\mathrm{Y} 537 \mathrm{~S}}$ and $\mathrm{MCF}^{\mathrm{Y} 537 \mathrm{~S}}$ (Fig. 4h) showed over $50 \%$ of the peaks called in MCF7 ${ }^{\mathrm{Y} 537 \mathrm{~S}}$ were common to those in SUM44-LTED ${ }^{\mathrm{Y} 537 \mathrm{~S}}$. Overlay of the common binding events with RNA-seq showed enrichment of genes associated with Hallmark pathways such as early $\left(p\right.$-value $=10^{-72}$, hypergeometric test $)$ and late $\left(p\right.$-value $=10^{-43}$, hypergeometric test) estrogen responsiveness, EMT transition $\left(p\right.$-value $=10^{-18}$, hypergeometric test), mTORC1 signaling ( $p$-value $=10^{-12}$, hypergeometric test), and fatty acid metabolism ( $p$-value $=10^{-9}$, hypergeometric test) (Supplementary Data 2). Nonetheless, differences between the cell lines highlight the influence of phenotypic nuances on the ESR1 function.
ESR1 $^{\text {wt }}$ and ESR1 ${ }^{\text {Y537C }}$ have altered genome-wide binding patterns. Two MCF7-LTED derivatives were sequenced, of which one harbored an ESR1 ${ }^{\text {Y537C }}$ (MCF7-LTED ${ }^{\text {Y537C) }}$ ) and the other ESR $1^{\text {wt }}$ (MCF7-LTED ${ }^{\mathrm{wt}}$ ) (as confirmed by ddPCR Supplementary Fig. 9a), suggesting LTED itself may not always select for mutations. Indeed, there are no previous reports of ESR1 mutations in LTED cells. Further interrogation of the whole-exome sequencing data from both MCF7-LTED models showed an increased mutational load in the MCF7-LTED ${ }^{Y 537 C}$ compared to the MCF7-LTED ${ }^{\text {wt }}$. However, no high impact mutations previously associated with AI resistance ${ }^{2}$ were evident in either cell line other than ESR1 Y537C (Supplementary Data 1). Immunoblotting showed that while key signaling pathways appeared similar between the LTED derivatives, expression of PGR differed significantly (Supplementary Fig. 9b). We therefore hypothesized that the mutant ESR1 $1^{Y 537 C}$ and ESR $1^{\text {wt }}$ controlled different ESR1 cistromes. To address this, genome-wide binding of ESR1 was assessed in both MCF7-LTED derivatives and the corresponding wt-MCF7. Assessment of the distribution of ESR1 binding showed increased occupancy at the promoter $(<1 \mathrm{~kb})$ in MCF7$\operatorname{LTED}^{\mathrm{wt}}\left(9.2 \%, p=10^{-94} \chi^{2}\right.$-test) and MCF7-LTED ${ }^{\mathrm{Y} 537 \mathrm{C}}(28.4 \%$, $p=0 \chi^{2}$-test) compared to wt-MCF7 (3.3\%). The converse was observed for the distal intergenic regions (Fig. 5a). To address this further, we used DiffBind and identified 4744 differential binding events between the MCF7-LTED ${ }^{\text {wt }}$ and wt-MCF7, 13,824 between MCF7-LTED ${ }^{\text {Y537C }}$ and wt-MCF7, and 11,018 between MCF7-LTED ${ }^{\text {wt }}$ and MCF7-LTED ${ }^{\mathrm{Y} 537 \mathrm{C}}$ (FDR <5\%) (Supplementary Fig. 9c, d). This suggested that the ESR1 ${ }^{\mathrm{Y} 537 \mathrm{C}}$ and ESR $1^{\text {wt }}$ in the MCF7-LTED cell lines control altered cistromes in comparison to wt-MCF7, but also differed between each other. Of interest, both LTED cell lines showed increased expression of GATA3, CDK1, RET, and ESR1 compared to the parental cell line (Fig. 5b). However, MCF7-LTED ${ }^{\mathrm{Y} 537 \mathrm{C}}$ showed increased expression of estrogen-regulated genes such as $P G R$ and TFF1 together with AREG, while MCF7-LTED ${ }^{\text {wt }}$ showed increased expression of BCL2 and XBP1 (Fig. 5b). K-means clustering of the ChIP-seq and RNA-seq data confirmed that the ESR1 Y537C mutation appeared to function "classically" in the absence of ligand compared to MCF7-LTED ${ }^{\mathrm{wt}}$. Noteworthy, both LTED derivatives enriched for pathways associated with PI3K/AKT/ mTORC compared to wt-MCF7 but differed in the down stream impact of these pathways when comparing clusters 1 and 3 (Fig. 5c-e).

We next assessed the metabolic capability of the cell lines, which was similar for both capacity and dependency on glutamine, and glucose (Fig. 5f). However, the MCF7-LTED ${ }^{\mathrm{wt}}$ showed higher dependency on fatty acids $(p<0.05$, one-way ANOVA and Tukey's test).

Finally, and in keeping with the SUM44-LTED, both MCF7LTED derivatives were highly migratory compared to wt-MCF7 (Fig. 5g).

In order to further delineate the dependency of the MCF7LTED $^{\mathrm{Y} 537 \mathrm{C}}$ on the mutant ESR1, we carried out a CRISPR-Cas9 reversion editing Y537C to Y537 $\left(\mathrm{ESR} 1^{\triangle 537 \mathrm{C}}\right.$ ) (Supplementary Fig. 10a, b). In keeping with our previous data, MCF7-LTED ${ }^{\mathrm{Y} 537 \mathrm{C}}$ showed ligand-independent growth. Contrastingly, MCF7-

Fig. 2 ESR1 ${ }^{Y 537 S}$ controls proliferation, EMT, and altered metabolism in SUM44-LTED. a Heatmap depicting the changes in gene expression from four identified clusters of genes that were significantly differentially expressed and bound by ESR1wt (wt-SUM44) or ESR1 ${ }^{\text {Y537S }}$ (SUM44-LTED). b Average log2 differences in ESR1 binding for all genes within each cluster during the course of adaptation to LTED. c Pathway analysis of the four clusters using GSEA. Sample labels represent: +E wkO = wt-SUM44, -E wk1 = 1 wk E-deprived SUM44, -E wk 20 = SUM44-LTED. d Metabolic dependency and capacity of wtSUM44 and SUM44-LTED on glutamine, fatty acid, and glucose using a Seahorse XFe96 analyzer. ( $n=4$ technical replicates). e Comparison of the migratory ability of wt-SUM44 and SUM44-LTED ( $n=8$ technical replicates). Error bars represent mean \pm SEM. Significance was assessed by Student's $t$ test. ${ }^{\star} p<0.05,{ }^{\star \star} p<0.01,{ }^{\star \star \star} p<0.001$ 
LTED $^{\triangle 537 C}$ and wt-MCF7 revealed limited proliferation in the absence of estrogen (Supplementary Fig. 10c). Furthermore, MCF7-LTED ${ }^{\Delta 537 C}$ switched to estrogen dependency and phenocopied the response of wt-MCF7 to fulvestrant (Supplementary Fig. 10d, e). Immunoblotting and RT-qPCR showed that MCF7-LTED ${ }^{\triangle 537 C}$ regain estrogen dependency for expression of a

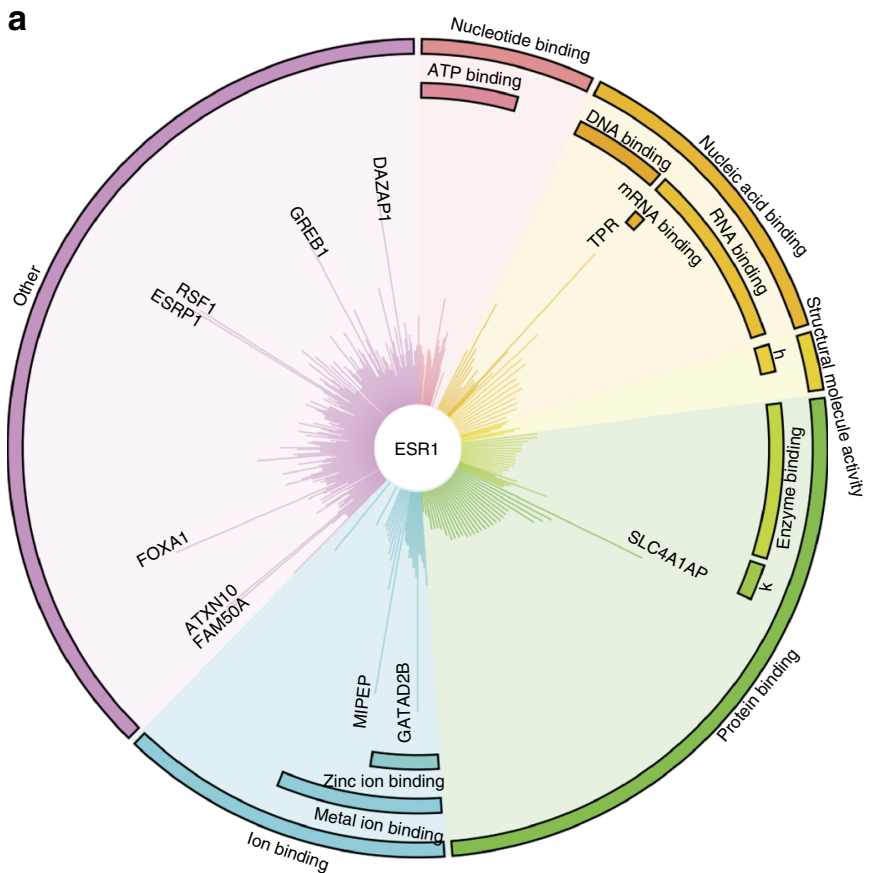

b

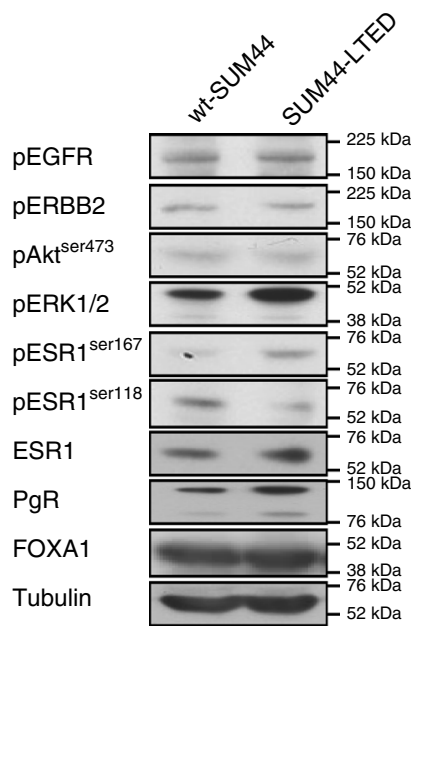

C

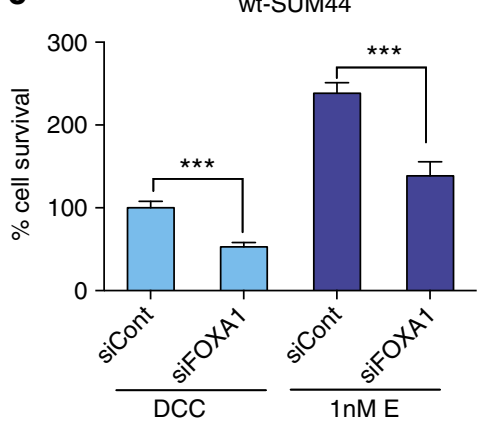

SUM44-LTED

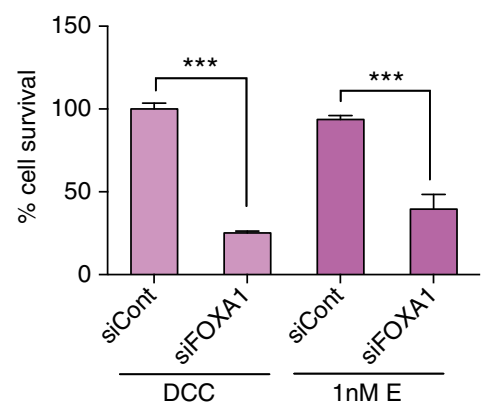

d

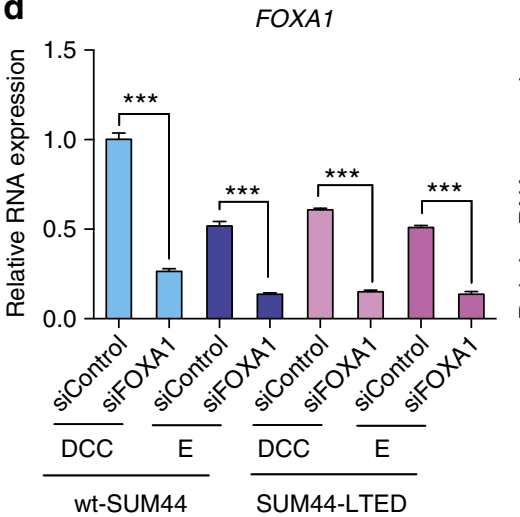

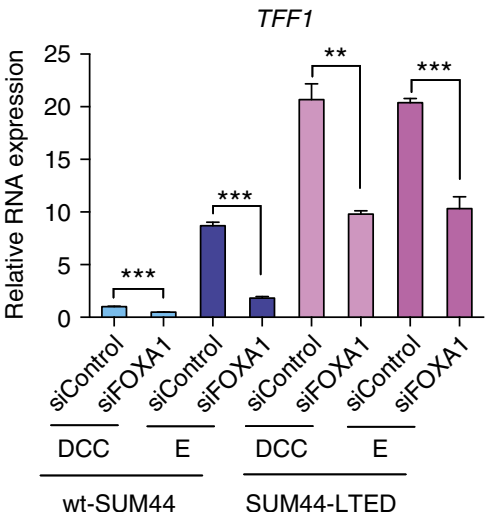

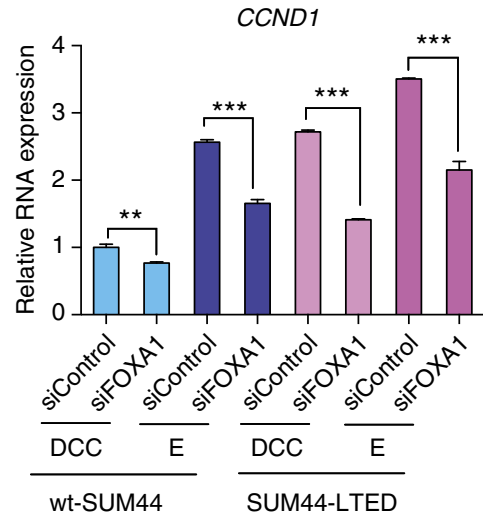

Fig. 3 Identification and functional analysis of the ESR1 ${ }^{\text {Y537S }}$ interactome. a MS-ARC depicting ESR1-RIME data conducted on SUM44-LTED (ESR1 ${ }^{\text {Y537S }}$ ) vS. wt-SUM44 (ESR1 ${ }^{\text {wt }}$ ) ( $n=3$ biological replicates). The ranking is based on SUM44-LTED/wt-SUM44 peptide (razor and unique) counts. The length of the line represents the number of identified peptides. The longer the line, the greater the interaction with ESR1 ${ }^{\mathrm{Y} 537 \mathrm{~S}}$ compared to ESRT ${ }^{\mathrm{wt}}$. The shorter cloud of lines shows the high degree of commonality in ESR1 binding proteins between both cell lines. $\mathbf{b}$ Immunoblotting showing alterations in expression of key protein markers previously associated with endocrine-resistant phenotypes. c Proliferation assays following siFOXA1 in wt-SUM44 and SUM44-LTED relative to siControl in the presence and absence of $\mathrm{E}$ (estradiol) ( $n=2$ biological experiments with eight technical replicates). $\mathbf{d}$ Expression of estrogenregulated genes, TFF1, and CCND1 following suppression of FOXA1 $\left(n=3\right.$ technical replicates). (error bars represent mean $\pm \mathrm{SEM},{ }^{\star} p<0.05,{ }^{\star \star} p<0.01$, ${ }^{\star \star \star} p<0.001$, significance was assessed by Student's $t$ test) 
target genes, PGR, TFF1, GREB1, and CTSD (Supplementary Fig. 10f, g). Taken together, these data show that the ESR $1^{\text {Y537C }}$ mutation is paramount for the ligand-independent phenotype of MCF7-LTED ${ }^{\text {Y537C }}$ cells.

ESR1 mutations show altered responses to endocrine therapy. One of the most clinically pressing questions relates to the sensitivity of ESR1 mutations to endocrine therapy. Cell lines were treated with escalating concentrations of 4-hydroxytamoxifen (4-OHT) or fulvestrant in the presence or absence of estrogen (Fig. 6a-c; Supplementary Fig. 8). In the absence of estrogen, both wt-MCF7 and wt-SUM44 showed little sensitivity to fulvestrant, as expected. SUM44-LTED and both MCF7-LTED derivatives were sensitive to fulvestrant in the absence of estrogen a

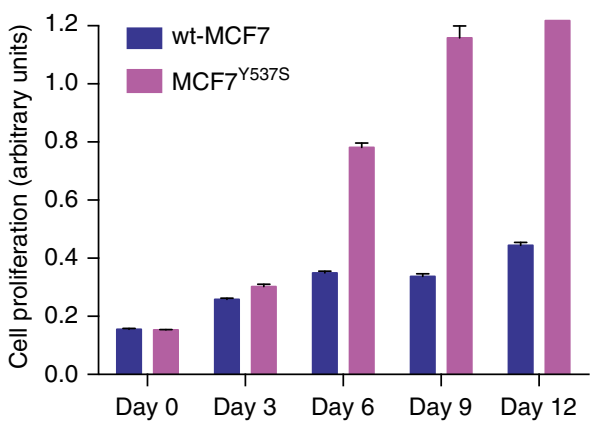

d
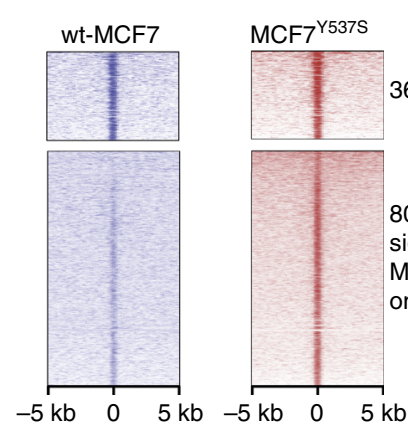

3602 common peaks

8094 peaks significant in only b

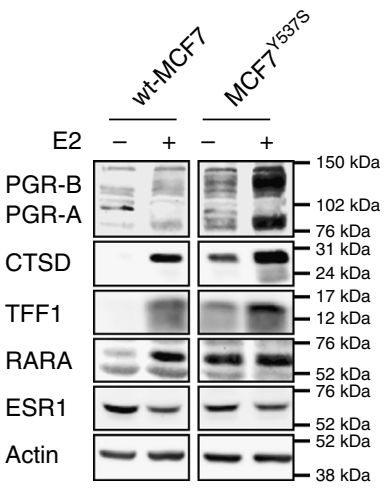

C

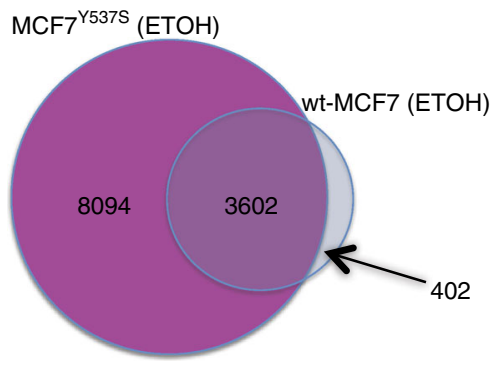

e

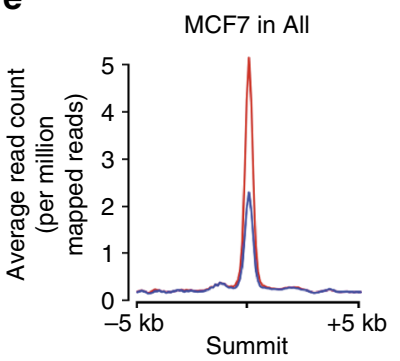

MCF7 inY537S

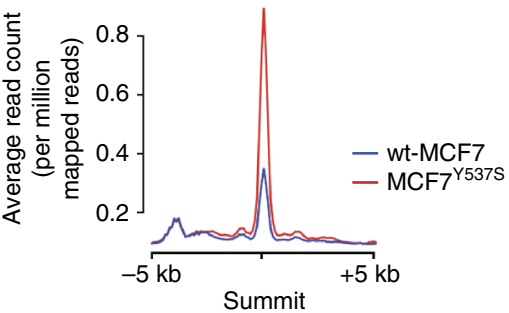

f

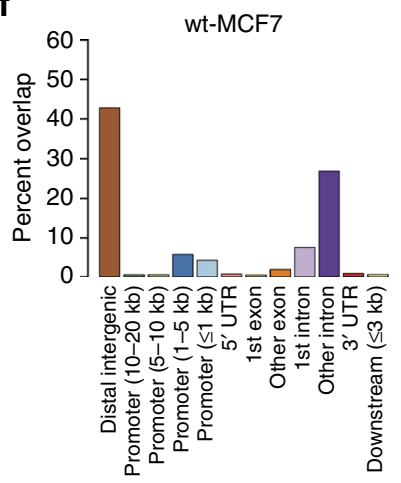

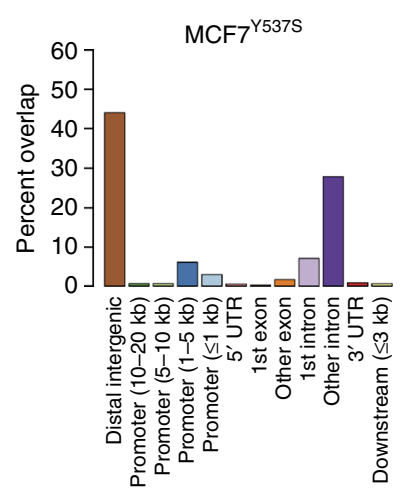

g

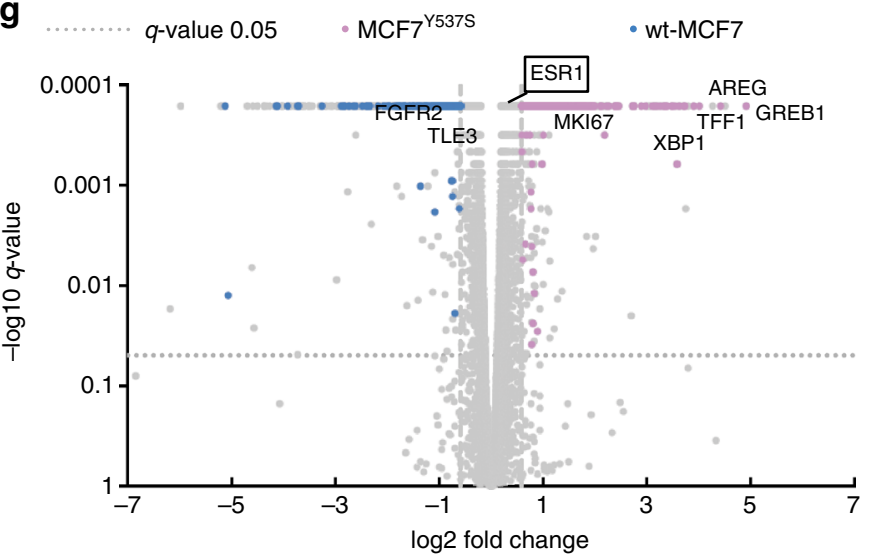

h

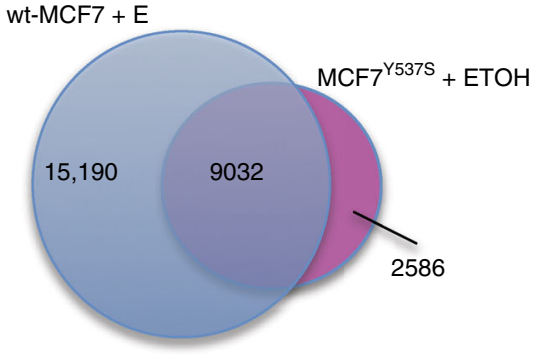

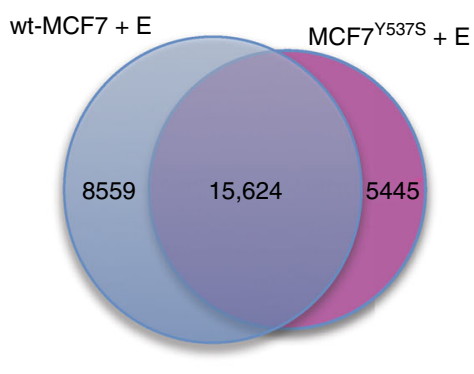

SUM44-LTED + ETOH

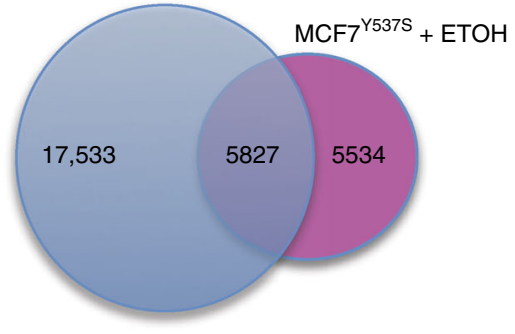


confirming ESR1 ligand independence, irrespective of mutation state. In the presence of estrogen, sensitivity to both 4-OHT and fulvestrant was reduced in the low concentration range in SUM44-LTED compared to wt-SUM44. However, while ESR1 $1{ }^{\mathrm{Y} 537 \mathrm{~S}}$ was not inhibited by 4-OHT, it was by fulvestrant. WtMCF7, MCF7-LTED ${ }^{\text {Y537C }}$, and MCF7-LTED ${ }^{\text {wt }}$ all showed similar sensitivity to 4-OHT. However, MCF7-LTED ${ }^{\mathrm{Y} 537 \mathrm{C}}$ in the presence or absence of estrogen showed greater sensitivity to fulvestrant compared to MCF7-LTED ${ }^{\text {wt }}$. The sensitivity of the MCF7-LTED ${ }^{\text {Y537C }}$ model to the antiproliferative effect of fulvestrant was further supported in vivo (Fig. 6d).

We subsequently assessed response to drugs inhibiting pathways associated with endocrine resistance such as mTORC (RAD001), ERK1/2 (U0126), and ERBB2/EGFR (lapatinib) ${ }^{2}$. SUM44 derivatives were resistant to the antiproliferative effects of lapatinib and U0126 and showed similar sensitivity to RAD001. The MCF7 derivatives revealed limited response to lapatinib. MCF7-LTED ${ }^{\mathrm{Y} 337 \mathrm{C}}$ and wt-MCF7 showed a similar response to RAD001 but not U0126, where MCF7-LTED ${ }^{\mathrm{Y} 537 \mathrm{C}}$ showed marked sensitivity in keeping with the increased levels of pERK1/2 in this cell line. The MCF7-LTED ${ }^{\text {wt }}$ showed little antiproliferative response to any of the agents tested, suggesting this cell line has a high degree of kinase plasticity (Supplementary Fig. 11a, b).

\section{Discussion}

Acquired resistance to endocrine therapy is a major clinical problem and the elucidations of pathways associated with relapse are of paramount clinical importance to facilitate improvement in treatment. While somatic mutations in ANDR have been strongly linked with lack of response to hormone therapy and/or agonist response to anti-androgens in prostate cancer, it is only recently that the importance of ESR1 mutations in BC has been reported (reviewed by Jeselsohn et al. ${ }^{7}$ ). In vitro studies using ectopic expression cassettes suggest that the most commonly found mutations, Y537S and D538G, confer ligand independence and exhibit reduced sensitivity to tamoxifen and fulvestrant ${ }^{11,12}$.

We describe for the first time the identification of naturally occurring ESR1 mutations in ESR1-positive BC cell lines. Importantly, we show that estrogen depletion selects for cells harboring ESR1 mutations, resulting in estrogen-independent growth and expression of the ESR1 transcriptome. We believe that normal culturing of $\mathrm{BC}$ cell lines in the presence of estrogen obviates the need for ESR1 mutations and that only with the strong selective pressure imparted by culturing in estrogendepleted medium are alternative growth pathways, including ESR1 mutations enriched. Furthermore, estrogen deprivation appears to be the primary point for enrichment, as ESR1 mutated cells did not appear to be augmented during acquisition of resistance to tamoxifen or fulvestrant in vitro. This observation is analogous to our recent clinical study in which ESR1 mutations in ctDNA of metastatic BC patients were found almost exclusively in patients that had become resistant to AI treatment ${ }^{10,13}$.
Additionally, treatment with fulvestrant in vitro appeared to enrich for the pre-existing Y537C mutation (MCF7-LTED-ICIR).

ChIP-seq analysis suggested that ESR $1^{\mathrm{Y} 537 \mathrm{~S}}$ functions in a ligand-independent manner, largely recapitulating the estrogenbound-ESR ${ }^{\text {wt }}$ cistrome, which was demonstrated by the fact that ER binding sites and their genomic distribution was overwhelmingly similar in wt-SUM44 and SUM44-LTED cells. The Y537S mutation lies near helix 12 (H12), which governs the ligand-regulated actions of ESR1 via AF-2. Recent studies have suggested that Y537S enables H12 to undergo a conformational change exposing the AF-2 cleft, facilitating recruitment of coregulators in the absence of hormone, leading to further stabilization of H12. In the same study, it was shown that Y537S also increased affinity for $\mathrm{AIB1}^{25}$. Assessment of the ESR1 ${ }^{\mathrm{Y} 537 \mathrm{~S}}$ interactome using RIME showed no increase in the association of the naturally occurring mutant ESR1 with AIB1, but did show increased association with FOXA1 and GREB1. One possible explanation for this difference is that the structural studies analyzed only the ESR1 LBD and nuclear receptor interacting domain of $\mathrm{AIB1}^{25}$ and thus cellular context was not explored.

Despite this compelling data, indicating the mutant ESR1 is sufficient to drive adaptation to estrogen deprivation, the cell lines, similar to clinical samples, are heterozygote for both wt and ESR1 mutant alleles. As such, we cannot conclusively differentiate between binding events due to wt and mutant ESR1, so it is possible that the wt allele predominates in LTED. However, there is no evidence in clinical samples that all ESR1 alleles are mutated in metastatic BC cases ${ }^{11,12,26-29}$. Moreover, MCF7 ${ }^{\mathrm{Y} 37 \mathrm{~S}}$ cells, generated by CRISPR-Cas9-mediated knockin mutagenesis, which are heterozygote for ESR $1^{\mathrm{Y} 537 \mathrm{~S}}$ and express both wt and Y537S mutant ESR1, show estrogen-independent recruitment of ESR1 and coactivators to ESR1 binding regions ${ }^{24}$. These cells demonstrate estrogen-independent expression of ESR1 target genes and grow in an estrogen-independent manner, validating the contribution of the Y537S mutation to estrogen independence when co-expressed with ESR1 ${ }^{\text {wt }}$.

A second caveat is the role of altered kinase signaling pathways that may arise from extended growth in estrogen-depleted culture conditions to generate LTED and post-translational changes that may impact on the resistance phenotype. Our own studies and those of others have shown that altered kinase signaling can lead to ligand-independent activation of ESR1 (reviewed by Ma et al. ${ }^{2}$ ). Furthermore, ectopic expression of AKT has been shown to alter the genome-wide binding pattern of ESR $1^{30}$ and that EGF induces a transcriptional program distinct from estrogen ${ }^{31}$. However, genomic profiling of SUM44-LTED cells harboring ESR 1 Y537S did not provide evidence for altered ESR1 binding patterns compared to wt-SUM44. Second, the CRISPR-Cas9derived MCF7 ${ }^{\mathrm{Y} 537 \mathrm{~S}}$ cells showed estrogen independence in the absence of prolonged culturing in estrogen-depleted conditions. Finally, CRISPR-Cas9 editing of the Y537C allele re-established estrogen dependence in MCF7-LTED ${ }^{\triangle 537 C}$ cells, demonstrating a requirement for the Y537C mutation for the estrogen

Fig. 4 Characterization of CRISPR-cas9-modified wt-MCF7 expressing ESR7 ${ }^{\text {Y537S }}$. a Viability assay showing MCF7Y537S proliferates in the absence of exogenous $\mathrm{E}$ compared to wt-MCF7 ( $n=6$ technical replicates and three biological replicates). Mean growth at day12 \pm SEM relative to day 0 . $\mathbf{b}$ Immunoblotting showing alterations in the expression of ESR1, PGR, CTSD, TFF1, and RARA. c Overlap between wt-MCF7 and MCF7 5375 ESR1 binding sites in the absence of $E$ and $\mathbf{d}$ corresponding heatmap. The heatmap depicts binding peak intensities that are common or different between the wt-MCF7 and MCF7 ${ }^{Y 537 S}$. The window represents $\pm 5 \mathrm{~kb}$ regions from the center of the binding event. e Comparison of the average read count between wt-MCF7 and MCF7 ${ }^{Y 537 S}$ in the absence of E showing peak affinity in both cell lines (left) and those binding sites only significant in MCF7 55375 (right) ( $q$-value < 0.05). $\mathbf{f}$ Bar chart showing the genomic distribution of ESR1 binding sites across the genome in both cell lines. $\mathbf{g} \vee$ olcano plot showing changes in gene expression by RNA-seq as a result of differential ESR1 ${ }^{\text {Y537S }}$ binding in MCF7 Y537S showing increased expression of estrogen-regulated and proliferationassociated genes. $\mathbf{h}$ Venn-diagrams showing intersect between wt-MCF7 and CRISPR generated MCF7 $5537 \mathrm{~S}$ ChIP-seq peaks in response to ethanol $(\mathrm{ETOH})$ or estradiol (E) and intersect between SUM44-LTED and MCF7 ${ }^{\text {Y537S }}$ in the absence of E 
independence. Taken together, our results support the notion that activating mutations in the ESR1 are sufficient for driving acquired resistance that does not necessitate changes in other signaling pathways.
Moreover, our in vitro data indicate that ESR1 ${ }^{\mathrm{Y} 537 \mathrm{~S} / \mathrm{C}}$ mutations are responsive to fulvestrant, as ESR1 protein expression was downregulated (Fig. 6c), although suppression of growth was less pronounced at low concentrations of the drug, indicating
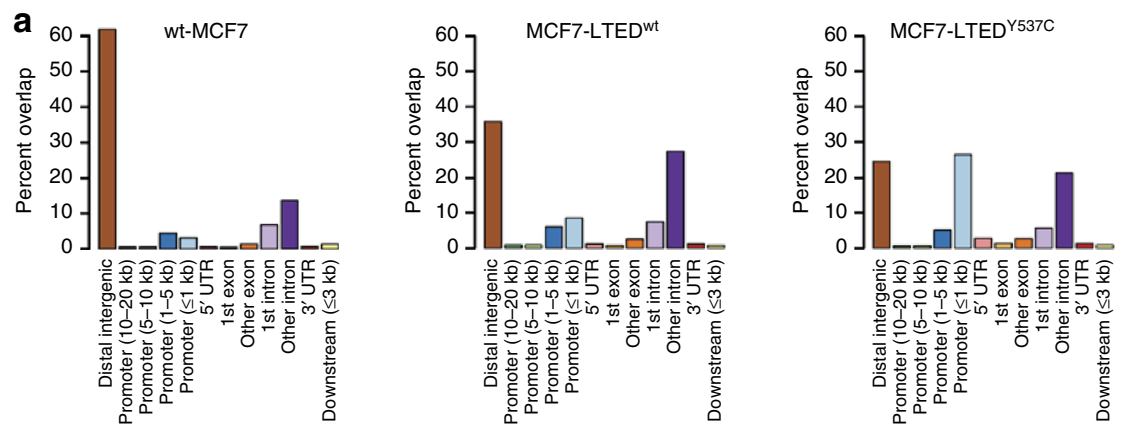

b

MCF7-LTED ${ }^{\text {wt }}$ vs. wt-MCF7

MCF7-LTED Y537C vs. wt-MCF7
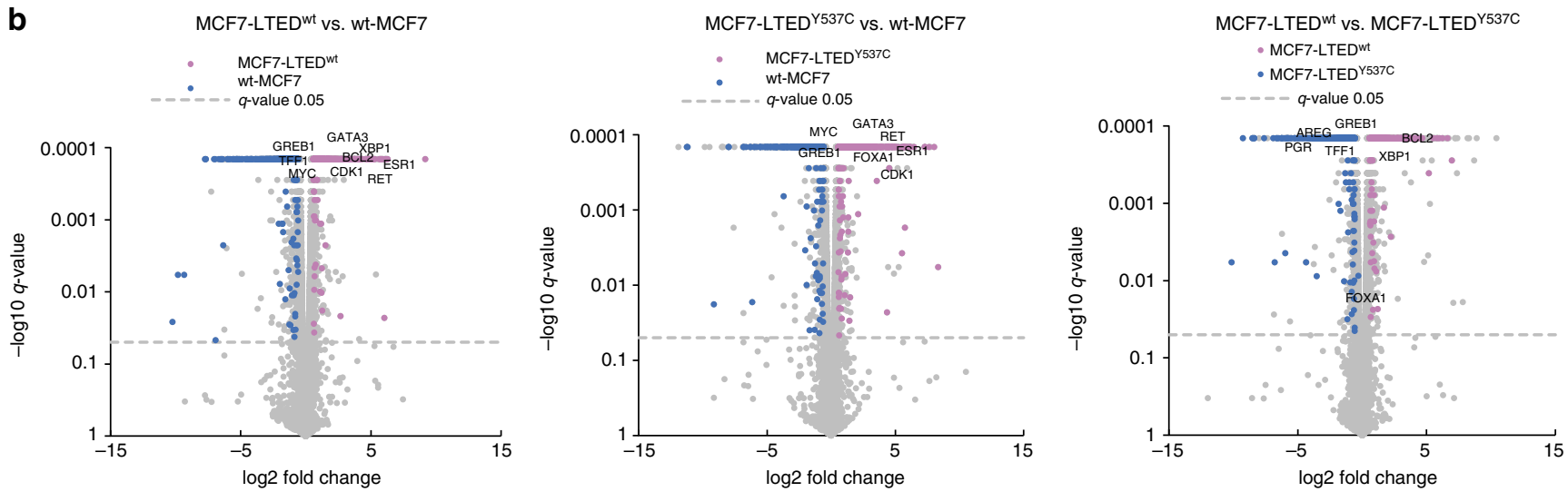

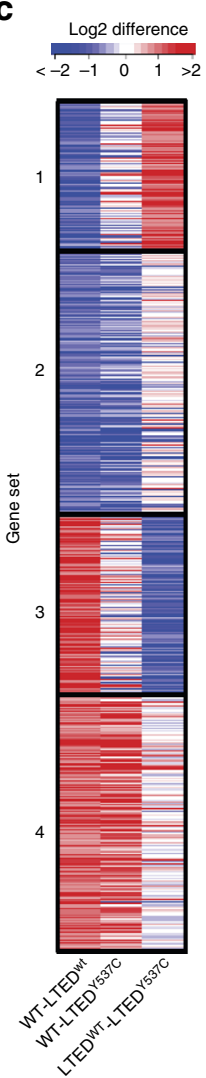

d
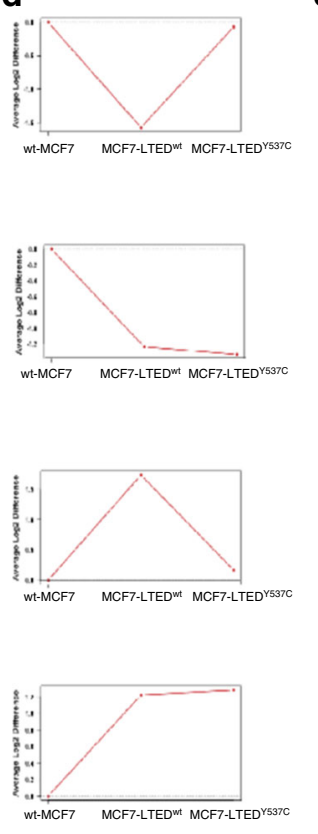

GENE SET NAME
HALLMARKESTROGEN_RESPONSE_EARLY
HALLMARK_ESTROGEN_RESPONSE_LATE

HALLMARK_UV_RESPONSE_DN

HALLMARK_TGF_BETA_SIGN
HALLMARK P53-PATHWAY

HALLMARK_WNT-BETA-CATENIN_SIGNALING

HALLMARK_EPITHELIAL MESENCHYMAL_TRANSITION

ALLMARK APOPTOSIS

GENE SET NAME

HALLMARK_ESTROGEN_RESPONSE_EARLY UNFOLDED_PROTEIN_RESPONS

HALLMARK_P53_PATHWAY
HALLMARK_TNFA_SIGNALING_VIA_NFKE

HALLMARK_MTORC1_SIGNALING

HALLMARK_GLYCOLYSIS
HALLMARK_INTERFERON_ALPHA_RESPONSE

HALLMARK_INTEREERON_ALPHA

HALLMARK_ANDROGEN_RESPONSE

GENE SET NAME

HALLMARK_TNFA_SIGNALING_VIA_NFKB

HALLMARK_ESTROGEN_RESPONSE_LATE

HALLMARK_UV_RESPONSE_UP

HALLMARK_APICAL_JUNCTIO

HALLMARK EPITHELIAL_MESENCHYMAL_TRANSITION

HALLMARK_HYPOXIA

HALLMARK_IL2_STAT5_SIGNALII

GENE SET NAME

HALLMARK_E2F_TARGETS

HALLMARK G GMM_CHECKPOIN

HALLMARK MTORC1 SIGNALING

HALLMARK_MTORC1SIGNALI
HALLMARK_GLYCOLYSIS

HALLMARK MYC_TARGETS $V$

HALLMARK_PEROXISOME

HALLMARK_P13K_AKT_MTOR_SIGNALING

HALLMARK_P53_PATHWAY f
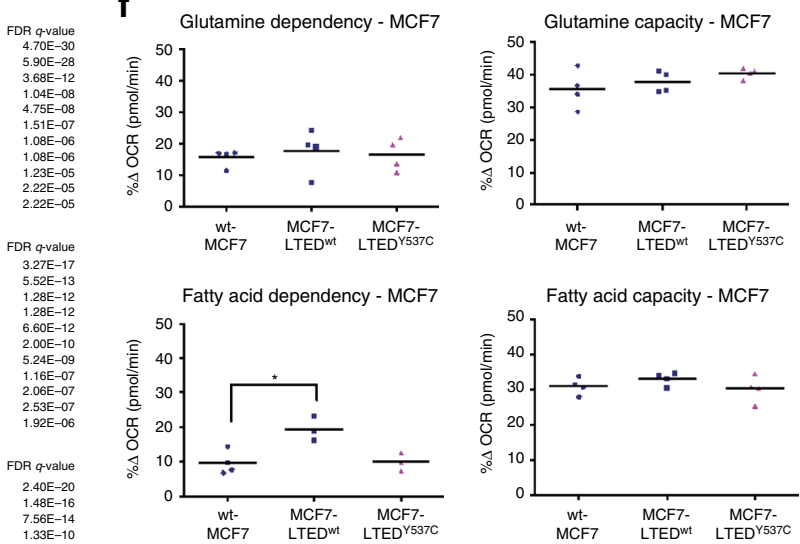

Glucose dependency - MCF7

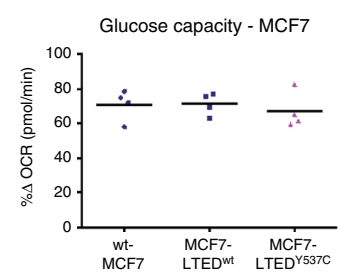

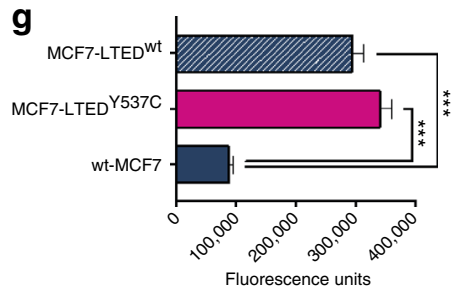


partial resistance of ESR $1^{\mathrm{Y} 537 \mathrm{~S}}$ but not ESR $1{ }^{\mathrm{Y} 537 \mathrm{C}}$. Nonetheless, at the predicted clinically achievable concentrations of fulves$\operatorname{trant}^{32,33}, \mathrm{ESR} 1^{\mathrm{Y} 537 \mathrm{~S}}$ was as equally sensitive as the ESR $1^{\mathrm{wt}}$. This is in keeping with our previous clinical data, which suggests patients harboring an ESR1 mutation show longer progressionfree survival when treated with fulvestrant vs. exemestane ${ }^{13}$. However, in contrast to Y537C, Y537S showed reduced sensitivity to 4-OHT. One explanation for these observations is that, 4-OHT causes Y537S to stabilize $\mathrm{H} 12$ by the formation of a hydrogen bond between $537 \mathrm{~S}$ and E380, effectively reducing the potency of the drug. In contrast, binding of fulvestrant disorders H12. As such, some of the new SERM/SERD agents with enhanced pharmacokinetics capable of increasing the dynamics of $\mathrm{H} 12$ may show increased potency against this mutation ${ }^{25}$.

Interestingly, MCF7-LTED ${ }^{\text {wt }}$ show evidence of reduced ESR1 activity, with lower expression of estrogen-regulated genes such as $P G R$ and increased expression of genes associated with antiapoptotic activity ${ }^{34}$. Unexpectedly, LTED cells expressing ESR1 $1^{\text {wt }}$ were also less sensitive to fulvestrant compared to ESR1 ${ }^{\mathrm{Y} 537 \mathrm{C}}$. One explanation is that these cells already have elevated kinase activities and are thus less dependent on ESR1, highlighting once again the complexity of cellular context as well as mutation status on response to endocrine therapy.

Recent genetic studies that have identified ESR1 mutations in metastatic, endocrine-resistant $\mathrm{BC}$ indicate that these mutations result from the selective pressure imposed by inhibition of ESR1 activity by hormonal therapies. The results presented here provide support for this hypothesis. The independent $\mathrm{BC}$ cell line models identified here also provide an important resource for studying the relative contribution of ESR1 mutations and alterations in other signaling pathways, that lead to endocrine resistance. Indeed, the genomic studies described herein provide support for the importance of kinase signaling cascades that have already been implicated in endocrine resistance by our studies, as well as those of other investigators. Our findings demonstrate that ESR1 mutations provide an important, albeit not the only driver of acquired endocrine resistance, concordant with the clinical observation that $\sim 20 \%$ of metastatic tumors harbor mutant ESR1. Using resistance models featuring ESR1 mutations and those that do not involve ESR1 mutations should prove to be valuable in aiding patient management, and for assessing new treatment approaches for endocrine-resistant BC. We and others will need to consider the presence and any phenotypic effects of these and possibly other acquired/selected mutations when using these derived cell lines for mechanistic or pharmacological studies and interpreting data from them.

\footnotetext{
Methods

Reagents. Following antibodies were used for immunoblotting: pESR1 ${ }^{\text {ser167 }}$ (CST cat-5587, 1:1000), pESR1 ${ }^{\text {ser118 }}$ (CST cat-2511, 1:1000), total-ESR1 (Santa Cruz sc8002, 1:800 or Novacastra (NCL-ER-6F11), 1:1000), total-FOXA1 (Abcam Ab23738, 1:1000) total-PGR (Novocastra NCL-L-PGR, 1:500 or Santa Cruz sc-538, 1:200), pERBB2 (CST-2243, 1:1000), total-ERBB2 (CST-4290, 1:1000), pEGFR (CST-3777, 1:1000), total-EGFR (CST-2232, 1:1000), pAKT ${ }^{\text {ser437 }}$ (CST-9271, 1:1000), total-AKT (CST-9272, 1:1000), pERK1/2 (Sigma-Aldrich, 1:2000), total-
}

ERK1/2 (CST-9102, 1:1000), TFF1 (Santa Cruz sc28925, 1:200), RARA (Abcam Ab39971, 1:1000), cathepsin D (CTSD) (Abcam Ab6313, 1:2000), actin (Abcam Ab6276, 1:10,000), and tubulin (Sigma T-9026, 1:2000). Secondary antibodies (horseradish peroxidase-linked, 1:2000) were obtained from Dako. For ChIP, the following antibodies were used: ESR1 (Santa Cruz sc543), CBP (Santa Cruz sc369), and FOXA1 (Abcam Ab23738). 17- $\beta$-estradiol (E) and 4-hydroxytamoxifen (4OHT) were purchased from Sigma-Aldrich and fulvestrant (ICI182780) from Tocris Bioscience.

Cell culture. Wt-MCF7, wt-HCC1428, wt-ZR75.1, and wt-SUM44 were purchased from the ATCC and Asterand. Cell lines were banked in multiple aliquots upon receipt to reduce risk of phenotypic drift and identity confirmed by short tandem repeats (STR) profiling. All cell lines were routinely screened for mycoplasma contamination. Wt cell lines were cultured in phenol red-free RPMI supplemented with $10 \%$ fetal bovine serum (FBS) and exogenous estradiol $(1 \mathrm{nM})$. The respective LTED derivatives were cultured, as previously described ${ }^{14,15}$ in phenol red-free RPMI supplemented with $10 \%$ dextran charcoal stripped FBS (DCC medium). ICI$\mathrm{R}$ and TAMR cell lines were cultured in their respective basal medium supplemented with $100 \mathrm{nM}$ fulvestrant (ICI182780) or $100 \mathrm{nM} 4-\mathrm{OHT}$. All experiments were performed under basal conditions unless otherwise stated.

Proliferation assays. Proliferation assays were performed as previously described for experiments involving drugs and siRNA studies ${ }^{14,15}$. In summary, cells were deprived of estrogen for $48-72 \mathrm{~h}$ prior to treatment with On-target plus siRNA for human-siFOXA1 or non-targeting pool (sicontrol) (Thermo scientific, Dharmacon). Knockdown efficacy was determined by qRT-PCR. For drug studies, cells were treated for 6 days with a medium change at day 3 , as previously described ${ }^{14}$ To analyze growth over time, cells were cultured as detailed above in DCC medium with or without estradiol and data recorded using an IncuCyte ZOOM live cell analyzer (Essen Biomedics). Three images per well were taken every $12 \mathrm{~h}$ over a 6 day period.

qRT-PCR. RNA was extracted using the RNeasy kit (Qiagen), quantified and reverse-transcribed with SuperScriptIII First Strand Synthesis System (Invitrogen) Taqman gene expression assays (Applied Biosystems) were used to quantify TFF1 (Hs00907239_m1 and Hs00170216_m1), PGR (Hs00172183_m1), GREB1 (Hs00536409_m1), CTSD (Hs00157201_m1), ESR1 (Hs00174860_m1), CCND1 (Hs00765553_m1), and the house-keeping genes FKBP15 (Hs00391480_m1) and GAPDH (Hs99999905_m1). The relative quantity was determined using $\Delta \Delta \mathrm{Ct}$, according to the manufacturer's instructions (Applied Biosystems).

Exome sequencing. Exome libraries were generated with SureSelect Human All Exon V5 kit and sequenced (paired-end $100 \mathrm{bp}$ ) on an Illumina HiSeq2500. Reads were aligned to GRCh37-lite-build37 using BWA mem (v0.7.12-r1039) ${ }^{35}$, sorted with samtools (v1.2) 36 and further processed using picard tools (http://picard. sourceforge.net) (v1.128) with default parameters. Single nucleotide variants (SNVs) were detected using VarScan v2.3.5 $5^{37}$ with default parameters (except --mpileup 1, --output-vcf) and wt cell samples as baseline. Multi-mapped reads were excluded and base alignment quality (BAQ) was turned off for pileup with samtools. To get high confidence somatic mutations, SNVs were filtered by using: (i) processSomatic of VarScan with empirically-derived criteria: minimum VAF in LTED cells: 0.10 , maximum VAF in wt: 0.05 , $p$-value $=0.07$; (ii) fpfilter.pl from VarScan together with bam-read count (--min-base-quality 15, --min-mappingquality 1) to reduce number of false positives. Variants were annotated using SnpEff v4.1 B (http://snpeff.sourceforge.net/SnpEff_manual.html). Mutations were annotated with Tier levels ${ }^{38}$ using BedTools v2.22.1 ${ }^{39}$. ascatNGS (https://github. $\mathrm{com} /$ cancerit/ascatNgs) was used to generate LogR and BAF values. Data have been deposited in the sequence read archive: BioProject ID PRJNA390496.

Ion torrent. DNA was amplified using Ion AmpliSeq Library Kit 2.0 (Life Technologies), digested, Ion Xpress.

Barcode adapters ligated and purified with Agencourt AMPure XP magnetic beads (Beckman Coulter). Libraries were quantified by qPCR using an Ion Library Quantification Kit (Life Technologies), templated on the Ion OneTouch2 System

\footnotetext{
Fig. $5 \mathrm{ESR} 1^{\mathrm{wt}}$ and $\mathrm{ESR} 1^{\mathrm{Y} 537 \mathrm{C}}$ regulate altered ESR1 cistrome. a Bar chart showing the genomic distribution of ESR1 binding sites across the genome in wtMCF7, MCF7-LTED ${ }^{\text {wt }}$, and MCF7-LTED ${ }^{Y 537 C}$ showing altered promoter $(\leq 1 \mathrm{~kb})$ and distal intergenic occupancy. b Volcano plots showing changes in gene expression by RNA-seq in MCF7-LTED Y537C, MCF7-LTED ${ }^{\text {wt }}$, and wt-MCF7. c Heatmap depicting the changes in gene expression of the four clusters comparing wt-MCF7 to MCF7-LTED Y537C, wt-MCF7 to MCF7-LTED wt, and MCF7-LTED ${ }^{\text {Y537C }}$ to MCF7-LTED ${ }^{\text {wt }}$. d Average log2 differences for all genes within each set for wt-MCF7, MCF7-LTED ${ }^{w t}$, and MCF7-LTED ${ }^{Y 537 C}$. e Pathway analysis of the four clusters using GSEA. Data were derived from $n=2$ biological replicates for ChIP-seq and $n=3$ biological replicates for RNA-seq. $\mathbf{f}$ Metabolic dependency and capacity of wt-MCF7, MCF7-LTED ${ }^{\text {wt }}$, and MCF7-LTEDY537C on glutamine, fatty acid, and glucose using a Seahorse XFe96 analyzer ( $n=4$ technical replicates). Significance was assessed by oneway ANOVA and Tukey's test. ${ }^{\star} p<0.05,{ }^{\star \star} p<0.01,{ }^{\star \star \star} p<0.001$. $\mathbf{g}$ Comparison of the migratory ability of wt-MCF7, MCF7-LTED ${ }^{\text {wt }}$, and MCF7$\operatorname{LTED}^{\text {Y537C }}\left(n=8\right.$ technical replicates). Data shown are mean \pm SEM. Significance was assessed by Student's $t$ test. ${ }^{\star} p<0.05,{ }^{\star \star} p<0.01,{ }^{\star \star \star} p<0.001$
} 
a
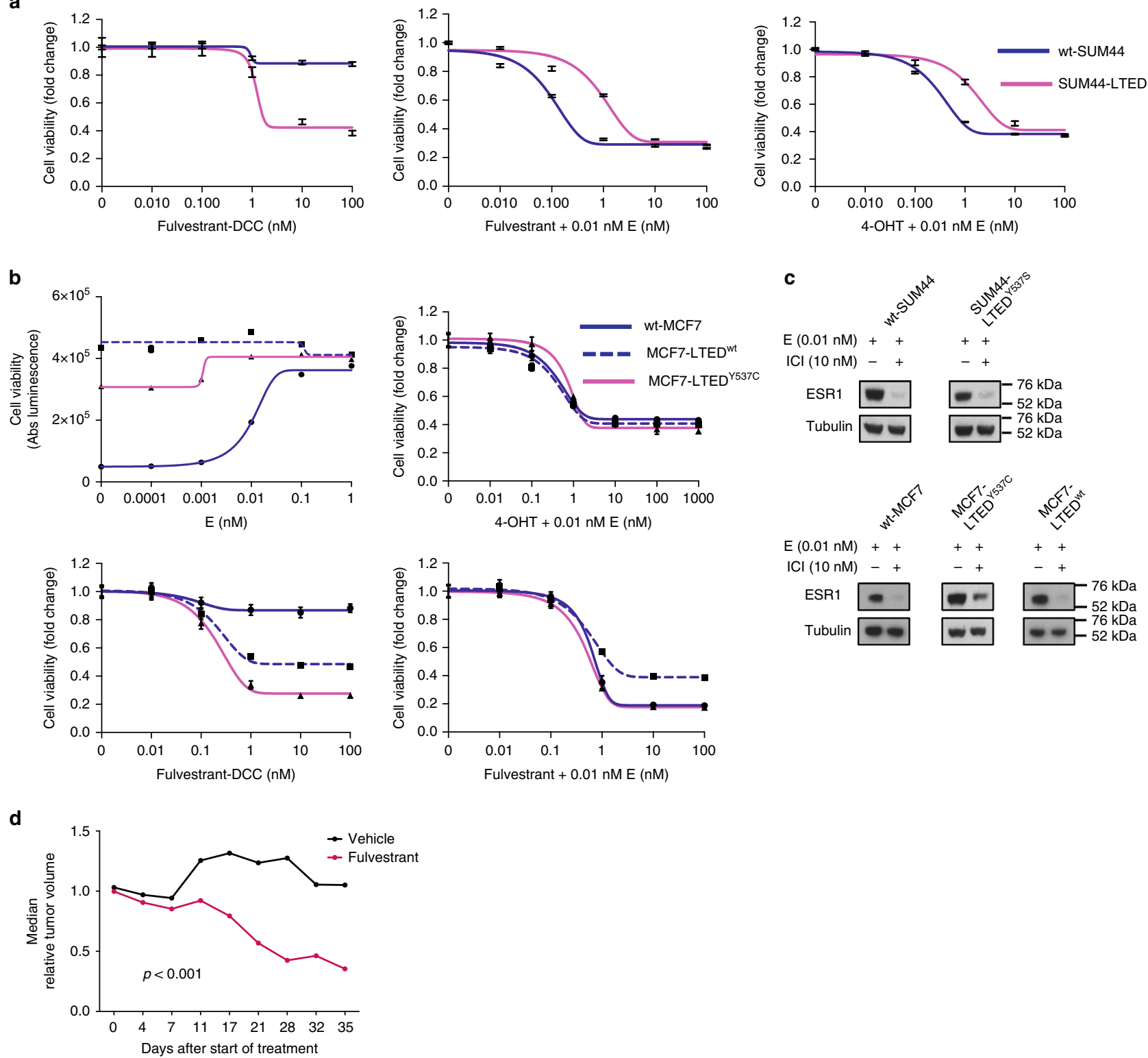

Fig. 6 Antiproliferative effect of endocrine therapy in ESR1 mutant and wt cell lines. a Proliferation assays assessing response of wt-SUM44 and SUM44LTED and b wt-MCF7, MCF7-LTEDWt, and MCF7-LTED Y537C to escalating concentration of fulvestrant \pm E (estradiol) and 4-OHT plus E (estradiol). c Treatment of wt-SUM44, SUM44-LTED ${ }^{\text {Y537S }}$, wt-MCF7, MCF7-LTED Y537C, and MCF7-LTED ${ }^{\text {wt }}$ with fulvestrant (10 nM) results in loss of ESR1 expression irrespective of mutation status ( $n=3$ biological replicates consisting of $n=8$ technical replicates). Data represent mean \pm SEM. $\mathbf{d}$ Xenograft models of MCF7-LTED Y537C in response to vehicle or fulvestrant. Data represent median fold change in tumor volume. Significance was assessed using an unpaired $t$ test

(Life Technologies) and sequenced on the Ion PGM System (Life Technologies). Reads were aligned by the PGM server with standard settings to the reference genome hg19, samtools v1.2 was used to calculate the on-target coverage.

IonReporter (v4.4) was used for mutation calling (parameters: data quality stringency $=12$, downsample to coverage $=4000, \mathrm{SNP} / \mathrm{InDel} / \mathrm{MNP}$ min cov each strand $=50, \mathrm{SNP} / \mathrm{InDel} / \mathrm{MNP}$ min variant score $=15, \mathrm{SNP} / \mathrm{InDel} / \mathrm{MNP} \min$ coverage $=250$, hotspot $\min$ variant score $=6$, hotspot min coverage $=150$ ). All mutations called were manually reviewed in Integrative Genomics Viewer (IGV) and included in the analysis if they had a VAF $\geq 1 \%$.

ddPCR. ddPCR assays for the ESR1 mutations Y537S and Y537C using Taqman probes were used as previously described ${ }^{10}$. Very-low-frequency mutations were only considered to be present if two or more FAM-positive droplets were detected in the total of the wt sample.

Cycle sequencing for validation. ESR1 mutations were validated by cycle sequencing by eurofins genomics (Eurofins). DNA was amplified using forward primer 5'- AAGTGGCTGCAGGGAGAGT-3' and reverse primer 5'TGGTGCATGATGAGGGTAAA-3'.

Fluorescence in situ hybridization. FISH probes hybridizing at 6q25 (ESR1) and chromosome-6 (CEN6) were purchased from Empire Genomics. Cell pellets were fixed in $4 \%$ paraformaldehyde and paraffin-embedded. Five-micron sections were subjected to the SwiftFISH rapid hybridization protocol (Empire Genomics), according to the manufacturer's instructions. Sections were mounted in DAPIcontaining Vectashield (Vector). FISH probes signals were analyzed using fluorescent microscope (Leica).

RNA-seq. Libraries were created after Ribo-zero rRNA Removal Kit (Illumina) using NEBNext Ultra Directional RNA (NEB) or Truseq Stranded Total RNA (Illumina) Library Prep Kit and sequenced using the HiSeq2500 (paired-end 100 bp v4 chemistry). Tophat (v2.1) and Cuffdiff (v2.2.1) ${ }^{40}$ using default parameters (GSE100075). K-means clustering was performed using the k-means function in the stats package in $R$. The number of clusters used was determined by the number 
of clusters generated in unsupervised clustering using hclust (method = complete) function in $\mathrm{R}$ with of a matrix of correlation-based distances using the Spearman method.

ChIP-seq. ChIP-qPCR and ChIP-seq were performed, as previously described ${ }^{14,41}$. Paired-end 50 bp ChIP-seq data were generated by rapid-mode HiSeq. Reads were aligned to the Human Reference Genome (assembly hg19) using BWA ${ }^{35}$ removing all reads with a quality score $<15$. Peaks were called using MACS2

(v2.1.0.20150420 $)^{42}$ with default parameters. Only binding events that occurred in two biological replicates were considered differential binding sites using Diffbind $\mathrm{v} 1.14 .5^{43}$ and $\mathrm{R}$ v3.2.1. Motif analysis was performed using centrimo (500 bp centered on summit of peak) (http://meme-suite.org/) (GSE100074). Bar charts were generated with ChIPseeker package in $\mathrm{R}^{44}$.

GSEA. Integration of RNA-seq and ChIP-seq diffBind data were carried out using GSEA, as previously described ${ }^{45}$. In summary, all genes assessed using RNA-seq were ranked and weighted by their mean Log2 fold change. Lists of genes that overlapped with regions showing significant differential binding were identified. These data were then analyzed using the GSEA v2.0.13 GSEA Pre-ranked tool. The default setting was applied. Finally, additional analysis of gene sets (e.g., overlaps between significant binding events and closest genes that are significantly differentially expressed) were performed using the Molecular Signature Database (http:// software.broadinstitute.org/gsea/msigdb/annotate.jsp) to compute overlaps with Hallmark gene sets that represent well-defined biological states or processes. Significance of overlap between gene sets was determined by hypergeometric test.

RIME and dimethyl labeling. RIME ${ }^{22}$ and stable isotope dimethyl labeling ${ }^{21}$ were performed, as previously described. The wt-SUM44 and SUM44-LTED were labeled with the medium and light isotope reagent, respectively. Labeled samples were pooled at an approximate 1:1 ratio, dried down and fractionated using $12 \mathrm{~cm}$ IPG strip pH 3-10, as previously described ${ }^{46}$. RIME and dimethyl label fractions were desalted (SUM SS18V, The Nest Group Inc) and run through LC-MS/MS using LTQ Velos Orbitrap MS. The data acquisition mode was set, as previously described $^{46}$. Raw data for RIME and dimethyl labeling were analyzed using MaxQuant 1.5.1.0 $0^{46,47}$. Search parameters were as previously described ${ }^{46}$. All proteomics data are deposited within the PRIDE database (PXD004807).

\section{Identification of mutation at protein level using ddMS2/PRM. ESR1-RIME} samples were subjected to ddMS2-PRM analysis in order to verify the presence of wt and mutated serine or cysteine in the SUM44-LTED and MCF7-LTED samples, respectively (Supplementary Data 3). The analysis was performed using a QExactive HF mass spectrometer (Thermo Scientific, Hemel Hempstead, UK). For each analysis, three biological replicates with two technical replicates were run. Heavy peptides were purchased from Thermo Fischer Scientific (PEPOTEC, grade 3). Reversed phase chromatography was performed on a Dionex UltiMate 3000 RSLC nano system (Thermo Fisher Scientific, Hemel Hempstead, UK) using an Acclaim PepMap100 C18 trap cartridge $(0.5 \mathrm{~mm}$ i.d. $\times 5 \mathrm{~mm}, 5 \mu \mathrm{m}$ bead size, $100 \AA$ pore size; loaded in a bi-directional manner). Peptides were resolved on a $75 \mu \mathrm{m} \mathrm{I}$. D. $50 \mathrm{~cm} \mathrm{C18}$ Easy-Spray packed emitter column $(2 \mu \mathrm{m}$ particle size; PepMap RSLC, Thermo Scientific, Hemel Hempstead, UK) over 90 min using a three-step gradient of $96: 4$ to $50: 50$ buffer $\mathrm{A}: \mathrm{B}(t=0 \min 4 \% \mathrm{~B}, 0.5 \min 4 \% \mathrm{~B}, 12.0 \mathrm{~min} 10 \% \mathrm{~B}$, $43.0 \min 25 \%$ B, $90.0 \mathrm{~min} 50 \%$ B) (buffer A: $2 \%$ acetonitrile/0.1\% formic acid; buffer B: $80 \%$ acetonitrile $/ 0.1 \%$ formic acid) at $250 \mathrm{nl}$ per min. Peptides were iodized by electrospray ionization using $1.8 \mathrm{kV}$ applied using the Easy-Spray ion Source. Sample was infused into the mass spectrometer directly from the packed emitter ( $5 \mu \mathrm{m}$ exit bore). The ion transfer tube was heated to $275^{\circ} \mathrm{C}$ and the S-lens set to $50 \%$. MS/MS were acquired using parallel reaction monitoring (PRM) and data-dependent (ddMS2) acquisitions based on a full FT-MS scan from 350 to $1850 \mathrm{~m} / z$ at 120,000 resolution, with a target automatic gain control (AGC) value of $3,000,000$ and a maximum injection time of $50 \mathrm{~ms}$. No internal lock mass calibrant was used. Eight PRM scans were triggered (FT-Orbitrap scans at 30,000 resolution, AGC target 2e5, $100 \mathrm{~ms}$ maximum injection time, normalized collision energy 35) if an ion from scheduled inclusion list was present. Then, the top five most intense ions were fragmented by higher energy collision-induced dissociation and dynamically excluded for $20 \mathrm{~s}$ (FT-Orbitrap scans at 30,000 resolution, AGC target 1e5, activation time $10 \mathrm{~ms}, 50 \mathrm{~ms}$ maximum injection time, normalized collision energy 28, selected first mass at $140 \mathrm{~m} / \mathrm{z}$ ). Precursor ions with unknown or single charge states were excluded from selection. Data analysis of raw MS/MS was carried out using Mascot V2.3 via Proteome Discoverer v1.4. Peak lists were searched against the human Uniprot FASTA database (20,305 sequences) containing the wt and mutant sequence. Spectra were searched for a match to fully-tryptic peptides with up to two missed cleavage sites. Search parameters were chosen as follows: serine/ threonine phosphorylation, protein $\mathrm{N}$-terminal acetylation, peptide $\mathrm{N}$-terminal glutamine to pyroGlu, and oxidation of methionines were all considered as variable modifications, whereas cysteine carbamidomethylation was selected as a fixed modification. Precursor ion mass tolerance was set to $15 \mathrm{ppm}$ for the first search, fragment ion mass tolerance for ion analyzed spectra was set to $0.02 \mathrm{Da}$. Resulting peptide and protein lists were grouped and validated using Scaffold v4 (Proteome Software Inc., Portland, OR). Protein identifications were automatically accepted if they contained at least two unique peptides assigned at $1 \%$ FDR. The raw data have been deposited in Passel (PASS01062).

Immunoblotting. Whole-cell extracts were generated from cells cultured under basal conditions or DCC medium with or without the addition of estrogen for comparative studies where noted. Equal amounts of protein were resolved by SDS-PAGE and subjected to immunoblot analysis. Antigen-antibody interactions were detected with ECL reagent (Amersham, UK) using the antibodies referred above.

\section{CRISPR-Cas9-mediated generation of the MCF7-LTED ${ }^{\triangle 537 C}$ cells. Gene} knockins for a modified ESR1 exon 8, encoding a wt open reading frame with silent mutations to facilitate PCR analysis, were made using CRISPR-Cas9-mediated homologous recombination in MCF7-LTED ${ }^{\mathrm{Y} 537 \mathrm{C}}$ cells. ESR1 gene targeting was carried out using CRISPR 4834093 (5'-GAGTGCTGAAATCCCTAGAA-3) cloned into a guide-RNA expression plasmid (a gift from George Church; Addgene plasmid \#41824), as described previously ${ }^{24}$. The target sequence for this CRISPR is located in intron 7, on the antisense strand, $73 \mathrm{nt}$ from the start of ESR1 Exon 8. For making the gene knockin, a previously described ESR1 exon 8 Y537S gene targeting donor construct ${ }^{24}$ was modified by site-directed mutagenesis to change codon 537 from Serine (TCT) to Tyrosine (TAT), as found in the wt sequence. Additional mutations, to destroy the PAM for CRISPR 4834093, were made by changing a run of four $\mathrm{C}$ nucleotides, located $77 \mathrm{nt} 5^{\prime}$ to the start of ESR1 Exon 8, to four $\mathrm{G}$ nucleotides. Genome editing, detection of gene targeting events, and sequence characterization of gene targeted alleles were carried out as described previously ${ }^{24}$, with the exception that following transfection, cells were recovered in full medium supplemented with 10\% FCS, and Exon 8 knockin clones identified through stochastic cloning.

Energy phenotype and Mito Fuel Flex analysis. SUM44 and MCF7 cells were plated at a confluency of $1.0 \times 10^{4}$ per well in a 96-well Seahorse cell culture microplates and incubated in a $5 \% \mathrm{CO}_{2}$ incubator at $37^{\circ} \mathrm{C}$ overnight. The next morning, culture media was replaced with $\mathrm{pH}$-adjusted $(\mathrm{pH}=7.4 \pm 0.1)$

bicarbonate-free DMEM with $10 \mathrm{mM}$ glucose, $1 \mathrm{mM}$ sodium pyruvate, and $2 \mathrm{mM}$ L-glutamine. The plate was then incubated at $37^{\circ} \mathrm{C}$ for $1 \mathrm{~h}$ in a non- $\mathrm{CO}_{2}$ incubator. For the Mito Fuel Flex test, oxygen consumption rates were measured using the Seahorse XF Mito Fuel Flex Test Kit (Agilent, 103260-100) on an XFe96 Analyzer. Cell numbers were normalized using CyQuant (Thermo Fisher, C35012).

Cell migration assay. Cells growing in basal media were washed several times with phenol red-free RPMI1640 containing 1\% DCC-FBS. A total of $2.5 \times 10^{4}$ cells were seeded into the upper chambers of Corning FluoroBlok 96-multiwell insert system plates (Corning, UK). The lower chambers were filled with RPMI1640 containing $1 \%$ DCC-FBS plus $100 \mathrm{ng} / \mathrm{ml}$ human recombinant EGF, as chemo-attractant, and plates were incubated at $37^{\circ} \mathrm{C}$. After $16 \mathrm{~h}$, the medium was removed from the lower chambers and wells were washed with PBS. PBS containing $1 \mu \mathrm{M}$ calcein AM (Invitrogen) was added to the lower chambers and the plates were incubated at $37^{\circ} \mathrm{C}$ for $30 \mathrm{~min}$. Fluorescence intensity was measured from the bottom of the plates using a $490 \mathrm{~nm}$ excitation filter and a $520 \mathrm{~nm}$ emission filter in a Victor X5 plate reader (PerkinElmer). Data are expressed as the mean of eight technical replicates.

Human tumor xenografts modeling relapse on Al therapy. In vivo studies were carried out in ovariectomized 8- to 12-week-old female BALB/c nude mice in accordance with Home Office Guidelines and approved by the Institute of Cancer Research Ethics Committee. Xenografts modeling patients resistant to AI were initiated by innoculating MCF7-LTED ${ }^{\mathrm{Y} 37 \mathrm{C}}\left(10^{7}\right)$ cells in basement membrane matrix (Matrigel; BD Biosciences) into the right flank of each animal. Once tumors reached $7 \mathrm{~mm}$ in size, they were size matched and mice treated with either $5 \mathrm{mg}$ per $\mathrm{kg}$ fulvestrant once per week or vehicle control. The study operator was blinded to treatment. Tumor growth was assessed twice weekly in both arms by caliper measurements of the two largest diameters. Volumes were then calculated according to the formula: $a \times b^{2} \times \pi / 6$, where $a$ and $b$ are orthogonal tumor diameters. Tumor volumes were then expressed as median relative fold change in volume at the start of treatment. At the end of study, data were available for seven animals in the control arm and nine animals in the fulvestrant treatment arm. Overall statistical differences between the treatment and control arms were calculated using an unpaired $t$-test.

Statistics analysis. Statistical methodologies pertinent to each method are held within the sections above.

Data availability. The data supporting the finding from this manuscript have been deposited as follows. Whole-exome sequencing has been deposited in the sequence read archive BioProject ID PRJNA390496. RNA-seq and ChIP-seq data have been deposited with the NCBI gene expression omnibus (GEO) (http://ncbi.nlm.nih. gov/geo/): ChIP-seq data for wt-MCF7, MCF7-LTED ${ }^{\text {wt }}$, MCF7-LTED $^{\text {Y537C }}$ wtSUM44 and SUM44-LTED (GSE100074), RNA-seq (GSE100075) for wt-MCF7, 
MCF7-LTED ${ }^{\text {wt }}$, MCF7-LTED ${ }^{\text {Y537C }}$, wt-SUM44 and SUM44-LTED, CRISPR-cas9 MCF7 ${ }^{\text {Y537S }}$ ChIP-seq and RNA-seq data (GSE78286) $)^{24}$. All proteomics data sets are deposited within the PRIDE database (PXD004807) or Passel (PASS01062) for targeted sequencing.

Received: 13 October 2016 Accepted: 20 October 2017

Published online: 30 November 2017

\section{References}

1. Green, K. A. \& Carroll, J. S. Oestrogen-receptor-mediated transcription and the influence of co-factors and chromatin state. Nat. Rev. Cancer 7, 713-722 (2007).

2. Ma, C. X., Reinert, T., Chmielewska, I. \& Ellis, M. J. Mechanisms of aromatase inhibitor resistance. Nat. Rev. Cancer 15, 261-275 (2015).

3. Early Breast Cancer Trialists' Collaborative Group. Aromatase inhibitors versus tamoxifen in early breast cancer: patient-level meta-analysis of the randomised trials. Lancet 386, 1341-1352 (2015).

4. Osborne, C. K. et al. Role of the estrogen receptor coactivator AIB1 (SRC-3) and HER-2/neu in tamoxifen resistance in breast cancer. J. Natl. Cancer Inst. 95, 353-361 (2003).

5. Miller, T. W., Balko, J. M. \& Arteaga, C. L. Phosphatidylinositol 3-kinase and antiestrogen resistance in breast cancer. J. Clin. Oncol. 29, 4452-4461 (2011).

6. Fuqua, S. A., Gu, G. \& Rechoum, Y. Estrogen receptor (ER) alpha mutations in breast cancer: hidden in plain sight. Breast Cancer Res. Treat. 144, 11-19 (2014).

7. Jeselsohn, R., Buchwalter, G., De Angelis, C., Brown, M. \& Schiff, R. ESR1 mutations-a mechanism for acquired endocrine resistance in breast cancer. Nat. Rev. Clin. Oncol. 12, 573-583 (2015).

8. Hortobagyi, G. N. et al. Correlative analysis of genetic alterations and everolimus benefit in hormone receptor-positive, human epidermal growth factor receptor 2-negative advanced breast cancer: results from BOLERO-2. J. Clin. Oncol. 34, 419-426 (2016).

9. Wang, P. et al. Sensitive detection of mono- and polyclonal ESR1 mutations in primary tumors, metastatic lesions, and cell-free DNA of breast cancer patients. Clin. Cancer Res. 22, 1130-1137 (2016).

10. Schiavon, G. et al. Analysis of ESR1 mutation in circulating tumor DNA demonstrates evolution during therapy for metastatic breast cancer. Sci. Transl. Med. 7, 313ra182 (2015).

11. Jeselsohn, R. et al. Emergence of constitutively active estrogen receptor-alpha mutations in pretreated advanced estrogen receptor-positive breast cancer. Clin. Cancer Res. 20, 1757-1767 (2014).

12. Toy, W. et al. ESR1 ligand-binding domain mutations in hormone-resistant breast cancer. Nat. Genet. 45, 1439-1445 (2013).

13. Fribbens, C. et al. Plasma ESR1 mutations and the treatment of estrogen receptor-positive advanced breast cancer. J. Clin. Oncol. 34, 2961-2968 (2016).

14. Ribas, R. et al. AKT antagonist AZD5363 influences estrogen receptor function in endocrine resistant breast cancer and synergises with fulvestrant (ICI182780) in vivo. Mol. Cancer Ther. 14, 2035-2048 (2015).

15. Ribas, R. et al. Identification of chemokine receptors as potential modulators of endocrine resistance in oestrogen receptor-positive breast cancers. Breast Cancer Res. 16, 447 (2014).

16. Ellis, M. J. et al. Whole-genome analysis informs breast cancer response to aromatase inhibition. Nature 486, 353-360 (2012).

17. Nik-Zainal, S. et al. Landscape of somatic mutations in 560 breast cancer whole-genome sequences. Nature 534, 47-54 (2016).

18. Sikora, M. J. et al. WNT4 mediates estrogen receptor signaling and endocrine resistance in invasive lobular carcinoma cell lines. Breast Cancer Res. 18, 92 (2016).

19. Carroll, J. S. et al. Chromosome-wide mapping of estrogen receptor binding reveals long-range regulation requiring the forkhead protein FoxA1. Cell 122, 33-43 (2005).

20. Shang, Y., Hu, X., DiRenzo, J., Lazar, M. A. \& Brown, M. Cofactor dynamics and sufficiency in estrogen receptor-regulated transcription. Cell 103, 843-852 (2000).

21. Boersema, P. J., Raijmakers, R., Lemeer, S., Mohammed, S. \& Heck, A. J. Multiplex peptide stable isotope dimethyl labeling for quantitative proteomics. Nat. Protoc. 4, 484-494 (2009).

22. Mohammed, H. et al. Endogenous purification reveals GREB1 as a key estrogen receptor regulatory factor. Cell Rep. 3, 342-349 (2013).

23. Hurtado, A., Holmes, K. A., Ross-Innes, C. S., Schmidt, D. \& Carroll, J. S. FOXA1 is a key determinant of estrogen receptor function and endocrine response. Nat. Genet. 43, 27-33 (2011).

24. Harrod, A. et al. Genomic modelling of the ESR1 Y537S mutation for evaluating function and new therapeutic approaches for metastatic breast cancer. Oncogene 36, 2286-2296 (2017).
25. Fanning, S. W. et al. Estrogen receptor alpha somatic mutations Y537S and D538G confer breast cancer endocrine resistance by stabilizing the activating function-2 binding conformation. eLife 5, e12792 (2016).

26. Zhang, Q. X., Borg, A., Wolf, D. M., Oesterreich, S. \& Fuqua, S. A. An estrogen receptor mutant with strong hormone-independent activity from a metastatic breast cancer. Cancer Res. 57, 1244-1249 (1997).

27. Li, S. et al. Endocrine-therapy-resistant ESR1 variants revealed by genomic characterization of breast-cancer-derived xenografts. Cell Rep. 4, 1116-1130 (2013).

28. Robinson, D. R. et al. Activating ESR1 mutations in hormone-resistant metastatic breast cancer. Nat. Genet. 45, 1446-1451 (2013).

29. Merenbakh-Lamin, K. et al. D538G mutation in estrogen receptor-alpha: a novel mechanism for acquired endocrine resistance in breast cancer. Cancer Res. 73, 6856-6864 (2013).

30. Bhat-Nakshatri, P. et al. AKT alters genome-wide estrogen receptor alpha binding and impacts estrogen signaling in breast cancer. Mol. Cell Biol. 28, 7487-7503 (2008).

31. Lupien, M. et al. Growth factor stimulation induces a distinct ER(alpha) cistrome underlying breast cancer endocrine resistance. Genes Dev. 24, 2219-2227 (2010).

32. McCormack, P. \& Sapunar, F. Pharmacokinetic profile of the fulvestrant loading dose regimen in postmenopausal women with hormone receptorpositive advanced breast cancer. Clin. Breast Cancer 8, 347-351 (2008).

33. Pritchard, K. I. et al. Results of a phase II study comparing three dosing regimens of fulvestrant in postmenopausal women with advanced breast cancer (FINDER2). Breast Cancer Res. Treat. 123, 453-461 (2010).

34. Gomez, B. P. et al. Human X-box binding protein-1 confers both estrogen independence and antiestrogen resistance in breast cancer cell lines. FASEB J. 21, 4013-4027 (2007).

35. Li, H. \& Durbin, R. Fast and accurate short read alignment with BurrowsWheeler transform. Bioinformatics 25, 1754-1760 (2009).

36. Li, H. et al. The Sequence Alignment/Map format and SAMtools. Bioinformatics 25, 2078-2079 (2009).

37. Koboldt, D. C. et al. VarScan: variant detection in massively parallel sequencing of individual and pooled samples. Bioinformatics 25, 2283-2285 (2009).

38. Mardis, E. R. et al. Recurring mutations found by sequencing an acute myeloid leukemia genome. N. Engl. J. Med. 361, 1058-1066 (2009).

39. Quinlan, A. R. \& Hall, I. M. BEDTools: a flexible suite of utilities for comparing genomic features. Bioinformatics 26, 841-842 (2010).

40. Trapnell, C. et al. Transcript assembly and quantification by RNA-Seq reveals unannotated transcripts and isoform switching during cell differentiation. Nat. Biotechnol. 28, 511-515 (2010).

41. Schmidt, D. et al. ChIP-seq: using high-throughput sequencing to discover protein-DNA interactions. Methods 48, 240-248 (2009).

42. Zhang, Y. et al. Model-based analysis of ChIP-Seq (MACS). Genome Biol. 9, R137 (2008).

43. Stark, R. \& Brown, G. D. DiffBind: Differential Binding Analysis of ChIP-Sec Peak Data Bioconductor http://bioconductor.org/packages/release/bioc/html/ DiffBind.html (2011).

44. Yu, G., Wang, L. G. \& He, Q. Y. ChIPseeker: an R/Bioconductor package for ChIP peak annotation, comparison and visualization. Bioinformatics 31, 2382-2383 (2015).

45. Mohammed, H. et al. Progesterone receptor modulates ERalpha action in breast cancer. Nature 523, 313-317 (2015).

46. Simigdala, N. et al. Cholesterol biosynthesis pathway as a novel mechanism of resistance to estrogen deprivation in estrogen receptor-positive breast cancer. Breast Cancer Res. 18, 58 (2016).

47. Cox, J. \& Mann, M. MaxQuant enables high peptide identification rates, individualized p.p.b.-range mass accuracies and proteome-wide protein quantification. Nat. Biotechnol. 26, 1367-1372 (2008).

\section{Acknowledgements}

This study was supported by Breast Cancer Now. We also acknowledge NHS funding to The Royal Marsden Hospitals NIHR Biomedical Research Centre and the generous funding from The Arthur Foundation in support of J.N.-B. S.A. and L.B. are funded by CRUK (C37/A18784), A.H. is supported by a PhD studentship from CRUK Imperial Centre. We also would like to thank the High-Throughput Genomics Group at the Wellcome Trust Centre for Human Genetics (funded by Wellcome Trust grant reference 090532/Z/09/Z) for the generation of the Sequencing data. Finally, we would like to thank the Tumor Profiling Unit within the Institute of Cancer Research.

\section{Author contributions}

The authors contributed to this work in different capacities described as follows. Concept: L.-A.M.; Generation of resistant models: L.-A.M., S.P., N.P., M.J.S; Experimental work: R.R., N.S., S.P., J.N.-B.; Droplet digital PCR: I.G.-M., C.F., Z.L., S.O.; Bioinformatics: N.T.; RNA-seq, ChIP-seq: E.S., R.R., N.S., J.C., W.Z.; Exome sequencing: R.R., Q.G., P.G.; Proteomics: N.S., A.B.; CRISPR: T.T., A.H., L.B., S.A.; Fluorescent in situ hybridization: V.M., M.H.; Metabolomics: M.-O.T., G.P.; Xenograft: A.T.; Manuscript 
and display item preparation: L.-A.M., R.R., N.S., S.A., E.S., M.D. All authors reviewed the prepared manuscript.

\section{Additional information}

Supplementary Information accompanies this paper at doi:10.1038/s41467-017-01864-y.

Competing interests: The authors declare no competing financial interests.

Reprints and permission information is available online at http://npg.nature.com/ reprintsandpermissions/

Publisher's note: Springer Nature remains neutral with regard to jurisdictional claims in published maps and institutional affiliations. (c) Open Access This article is licensed under a Creative Commons Attribution 4.0 International License, which permits use, sharing, adaptation, distribution and reproduction in any medium or format, as long as you give appropriate credit to the original author(s) and the source, provide a link to the Creative Commons license, and indicate if changes were made. The images or other third party material in this article are included in the article's Creative Commons license, unless indicated otherwise in a credit line to the material. If material is not included in the article's Creative Commons license and your intended use is not permitted by statutory regulation or exceeds the permitted use, you will need to obtain permission directly from the copyright holder. To view a copy of this license, visit http://creativecommons.org/ licenses/by/4.0/.

(C) The Author(s) 2017 